\title{
Triacylglycerol synthesis by PDAT1 in the absence of DGAT1 activity is dependent on re-acylation of LPC by LPCAT2
}

Jingyu X $\mathrm{U}^{1}$, Anders S Carlsson ${ }^{2}$, Tammy Francis ${ }^{1}$, Meng Zhang ${ }^{3}$, Travis Hoffman ${ }^{1}$, Michael E Giblin ${ }^{1}$ and David C Taylor ${ }^{1,4^{*}}$

\begin{abstract}
Background: The Arabidopsis thaliana dgat1 mutant, AS11, has an oil content which is decreased by 30\%, and a strongly increased ratio of 18:3/20:1, compared to wild type. Despite lacking a functional DGAT1, AS11 still manages to make $70 \%$ of WT seed oil levels. Recently, it was demonstrated that in the absence of DGAT1, PDAT1 was essential for normal seed development, and is a dominant determinant in Arabidopsis TAG biosynthesis.

Methods: Biochemical, metabolic and gene expression studies combined with genetic crossing of selected Arabidopsis mutants have been carried out to demonstrate the contribution of Arabidopsis PDAT1 and LPCAT2 in the absence of DGAT1 activity.

Results: Through microarray and RT-PCR gene expression analyses of AS11 Vs. WT mid-developing siliques, we observed consistent trends between the two methods. FAD2 and FAD3 were up-regulated and FAE1 downregulated, consistent with the AS11 acyl phenotype. PDAT1 expression was up-regulated by ca 65\% while PDAT2 expression was up-regulated only 15\%, reinforcing the dominant role of PDAT1 in AS11 TAG biosynthesis. The expression of LPCAT2 was up-regulated by 50-75\%, while LPCAT1 expression was not significantly affected. In vitro LPCAT activity was enhanced by $75-125 \%$ in microsomal protein preparations from mid-developing AS11 seed vs WT. Co-incident homozygous knockout lines of dgat1/lpcat2 exhibited severe penalties on TAG biosynthesis, delayed plant development and seed set, even with a functional PDAT1; the double mutant dgat1/Ipcat1 showed only marginally lower oil content than AS11.

Conclusions: Collectively, the data strongly support that in AS11 it is LPCAT2 up-regulation which is primarily responsible for assisting in PDAT1-catalyzed TAG biosynthesis, maintaining a supply of PC as co-substrate to transfer sn-2 moieties to the sn-3 position of the enlarged AS11 DAG pool.
\end{abstract}

Keywords: dgat1 mutant AS11, LPCAT1, LPCAT2, PDAT1, Oil biosynthesis Seed lines from Nottingham Arabidopsis Stock Centre WT (ecotype Columbia-0), dgat1, AS11 (CS3861), A7 (SALK_039456), Ipcat1 (SALK_123480), Ipcat2 (SAlL_357_H01) (all in a Columbia background)

\section{Background}

Triacylglycerols (TAGs) are the major storage lipids which accumulate in developing seeds, flower petals, anthers, pollen grains, and fruit mesocarp of a number of plant species [1,2]. TAGs are thought to be not only the major energy source for seed germination but also

\footnotetext{
* Correspondence: David.Taylor@nrc-cnrc.gc.ca

'National Research Council of Canada, Plant Biotechnology Institute, 110

Gymnasium Place, Saskatoon, SK S7N OW9, Canada Full list of author information is available at the end of the article
}

essential for pollen development and sexual reproduction in many plants $[3,4]$. In oilseeds, TAG bioassembly is catalyzed by the membrane-bound enzymes of the Kennedy pathway that operate in the endoplasmic reticulum [4]. The biosynthesis of TAGs is catalyzed by the sequential acylation of the glycerol backbone involving three acyltransferases: glycerol-3-phosphate acyltransferase (GPAT), lyso-phosphatidic acid acyltransferase (LPAAT) and diacylglycerol acyltransferase (DGAT). DGAT catalyses the final acylation of DAG to give
C Biomed Central 
TAG, which has been suggested to be the rate-limiting step in plant lipid accumulation.

In the traditional Kennedy pathway DGAT was thought to be the only enzyme that is exclusively committed to TAG biosynthesis using acyl-CoA as its acyl donor. The first DGAT gene was cloned from mouse and is a member of the DGAT1 family, which had high sequence similarity with sterol: acyl-CoA acyltransferase [5].

We had previously characterized an EMS-induced mutant of Arabidopsis, designated AS11, which displayed a decrease in stored seed TAG, delayed seed development, and an altered fatty acid composition [6]. We analyzed WT vs. AS11 lipid pools and Kennedy pathway enzyme activities in fractions isolated from green mid-developing seed, and performed parallel labeling of intact seeds at this developmental stage, with $\left[{ }^{14} \mathrm{C}\right]$ acetate. We found that compared to WT, there was an increase in all fatty acids in the DAG pool of AS11 seeds at mid-development, and, to a lesser extent, an associated backup of fatty acids in the PC pool. DAG was elevated from $1 \%$ in WT to $10-12 \%$ in AS11 and PC pools were elevated from about $2 \%$ in WT, to $8-12 \%$ in AS11. Cell-free fractions from WT and AS11 green seeds at mid-development were compared for their ability to incorporate [ $\left.{ }^{14} \mathrm{C}\right]-18: 1-\mathrm{CoA}$ into glycerolipids in the presence of G-3-P. Proportions of labeled LPA and PA formed during the incubation period were similar in WT vs AS11, indicating that the activities of the Kennedy pathway enzymes GPAT and LPAAT (EC 2.3.1.51) were relatively unaffected in the AS11 mutant. However, the proportion of labeled TAG was much lower and that of DAG was much higher in AS11. The TAG/DAG ratio was therefore consistently 3-5-fold lower in $A S 11$ compared to WT at all developmental stages (early-, mid- and late development). Furthermore, the ratio of 18:3/20:1 dramatically increased about 7-10 fold [6].

Cumulatively, this data suggested a lesion in DGAT1 which was subsequently demonstrated upon cloning the mutated gene from AS11. There is an $81 \mathrm{bp}$ in-frame insertion consisting entirely of exon 2 in the transcript from AS11. The exon 2 in the repeat is properly spliced, thus the alteration of the transcript does not disturb the reading frame. However, this additional exon 2 sequence in the AS11 transcript would result in an altered DGAT protein with a 27 amino acid insertion $\left({ }^{131}\right.$ SHAGLFNLC VVVLIAVNSRLIIENLMK ${ }^{157}$ ) [7]. It is important to note that two other labs independently and simultaneously cloned the A. thaliana DGAT1 $[8,9]$.

Studies manipulating the expression of DGAT1 followed: We demonstrated that expression of the Arabidopsis DGAT1 cDNA in a seed specific manner in the AS11 mutant restored wild type levels of TAG and VLCFA content. The acyl distribution, specifically, the sn-3 composition of the TAGs, was also restored to WT proportions. Furthermore, overexpression of the Arabidopsis DGAT1 in wild type plants led to an increase in seed oil content and seed weight [10]. Over the past 10 years, DGAT1 expression has been genetically manipulated to produce Brassica napus prototypes containing increased seed oil $[11,12]$.

A second family of $D G A T$ genes (DGAT2), first identified in the oleaginous fungus Morteriella ramanniana, has no sequence similarity with DGAT1 [13]. A human $D G A T 2$ and plant DGAT2s from tung and castor were subsequently identified by Cases et al. [14], Shockey et al. [15] and Kroon et al. [16], respectively. The putative DGAT2 from Arabidopsis has been studied by several labs including ours; functional expression in yeast has not been successful, and therefore whether it is a true functioning DGAT is still in question. Notably, an Arabidopsis dgat2 knockout mutant has a wild-type seed oil content and fatty acid composition [17].

TAG can also be formed by an acyl-CoA-independent enzyme, phosphatidylcholine: diacylglycerol acyltransferase (PDAT), in which the transfer of an acyl group from the $s n-2$ position of $\mathrm{PC}$ to the $s n-3$ position of DAG yields TAG and sn-1 lyso-PC [18,19]. During the exponential growth phase in yeast, PDAT1 is a major determinant in TAG synthesis. In Arabidopsis, two close homologs to the yeast $P D A T$ gene have been identified: PDAT1 At5g13640 and PDAT2 At3g44830 [20]. Mhaske et al. [21] isolated and characterized a knockout mutant of Arabidopsis which has a T-DNA insertion in the PDAT1 locus At5g13640 (PDAT1, EC 2.3.1.158). Lipid analyses were conducted on this mutant to assess the contribution of PDAT1 to seed lipid biosynthesis; surprisingly, and in contrast to the situation in yeast, the oil content and composition in seeds did not show significant changes in the mutant. At the time, these results were interpreted to indicate that PDAT1 activity as encoded by At5g13640 is not a major determining factor for TAG synthesis in Arabidopsis seeds.

Nonetheless, because the Arabidopsis DGAT1 mutant AS11 shows only a $30-35 \%$ decrease in oil content $[6,9]$, it was apparent that other enzymes must contribute to oil synthesis in the developing seed [22]. Thus, an examination of the contribution of DGAT2, PDAT2 or PDAT1 to oil deposition in an AS11 background was studied by performing double mutant crosses with $A S 11$ [17]. While the dgat2-ko line has no oil phenotype, homozygous double mutants from cross of AS11 with dgat2-ko mutant showed an oil fatty acid profile similar to $A S 11$. The same pattern was observed with the pdat2-ko mutant alone and in crosses of the pdat2-ko mutant with $A S 11$. In contrast, while the pdat1-ko has no oil or fatty acid composition phenotype, crosses of the pdat1-ko with AS11 were embryo-lethal in the 
double homozygous condition; only heterozygous lines produced by having expression of the pdat1 or dgat1 gene only partially inhibited using RNAi, allowed an examination of the double mutants. These detailed studies resulted in the finding that DGAT1 and PDAT1 have overlapping functions in both embryo development and TAG biosynthesis in the developing seed and in pollen. When DGAT1 is compromised in AS11, it is PDAT1, and not DGAT2 or PDAT2, that is responsible for the remaining $65-70 \%$ of TAG which is synthesized. This finding suggested a major, perhaps dominant role of PDAT1 in this process [17].

Recently, a castor bean-specific PDAT, PDAT1-2, was cloned and found to be highly expressed in developing seeds and localized in the ER, similar to the castor FAH12 hydroxylase. Transgenic Arabidopsis co-expressing the castor PDAT1-2 and FAH12 showed enhanced ricinoleate accumulation to up to $25 \%$ in TAGs (compared to $17 \%$ in FAH12 -only transgenics) [23,24]. This study suggests that specialized PDATs may play a significant role in channeling PC-synthesized unusual fatty acids such as ricinoleic (from castor), or epoxy fatty acids (e.g. from Vernonia galamensis), into TAGs.

The discovery of the critical role PDAT1 has in TAG synthesis [17] suggested the importance of re-acylation of lysophospholipids and especially LPC, since it is produced as a result of PDAT1 activity. We hypothesized that in a situation where the responsibility for TAG synthesis is shifted to PDAT1 such as in the AS11 dgat1 mutant, profound changes in activity of re-acylation enzymes would be evident. As candidates of genes coding such enzymes, we proposed the two Arabidopsis genes LPLAT1 (At1g12640) and LPLAT2 (At1g63050), characterized by Ståhl et al. [25] as possessing broad specificity lysophospholipid acyltransferase activity. Although both of the genes to some extent could acylate a range of different lysophospholipids, they were shown to have a strong preference towards LPC. For this reason we chose to name the two genes LPCAT1 (At1g12640) and LPCAT2 (At1g63050), in the current study.

Here we report the further genetic and biochemical characterization of the AS11 dgat1 mutant. During the course of microarray and qRT-PCR studies of AS11 vs WT gene expression in mid-developing siliques, we found that LPCAT2, encoding acyl-CoA:lysophosphatidylcholine acyltransferase 2 (EC 2.3.1.23), was up-regulated while LPCAT1 was not affected. By a series of biochemical studies and key crosses of AS11 with either lpcat 1 or lpcat 2 knockout mutants, we determined that LPCAT2 (and much less so LPCAT1) is most critical for TAG synthesis in the AS11 mutant, primarily to maintain the PC pool for TAG assembly primarily catalyzed by PDAT1.

\section{Results and discussion}

Summary of the AS11 mutant developmental and oil phenotypes

The AS11 mutant line was about one week behind WT in bolting and entering the generative phase and thus, under our growing conditions, AS11 seed set was also delayed to four weeks post-anthesis instead of three, as typically observed in WT. For comparison, we studied another DGAT1 mutant, which we designated $A 7$, which is a homozygous SALK line (Salk 039456) with a TDNA insertion in the last exon of the same DGAT1 gene (At2g19450). A7 shows a developmental delay similar to that exhibited by AS11 (Additional file 1: Figure S1).

Using protein fractions prepared from WT and AS11 mid-developing siliques containing embryos at middevelopment (Stages 6-8 as designated in the "Methods" section) we were able to determine the relative changes of TAG assembly activity in the mutant line. TAG synthesis capacity was measured in WT and AS11 lines with ${ }^{14} \mathrm{C}$-labeled diolein and unlabeled oleoyl-CoA as co-substrates; the ${ }^{14} \mathrm{C}$-labeled triolein product was measured by radio-HPLC as described previously [26]. As shown in Figure 1, by stages 7-8 there was a $35 \%$ decrease in the acylation of radiolabeled DAG in AS11, a finding which was strongly correlated with the $c a 30 \%$ reduction in oil content in mature AS11 seed $[6,10]$.

\section{Heterologous expression of mutated Arabidopsis DGAT1s

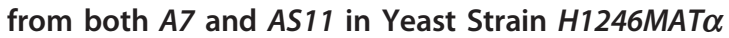

The altered fatty acid and low TAG phenotype in AS11 seed raised questions as to how the AS11 mutant still manages to make $65-70 \%$ of WT levels of seed oil. Because $A S 11$ has a reduced TAG phenotype, it was essential to determine whether the DGAT1 in AS11 was merely mutated and exhibited reduced activity as we initially suggested [6], or whether it is, in fact, non-functional. This has not heretofore been confirmed. The importance of doing so will become apparent in the PCR assessment of the dgat1 transcript in both AS11 and in some genetic crosses performed in the current study, and discussed below. The cDNA from the AS11 (with a $81 \mathrm{bp}$ in-frame repeat insertion of the second exon) was cloned into a yeast expression vector pYES2.1 under the control of the galactose-inducible GAL1 promoter, and the construct was used to transform a yeast mutant strain

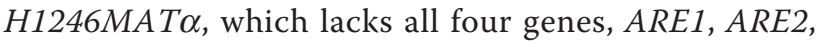
DGAT1 and LRO1, which were found to contribute to TAG synthesis [27]. This yeast mutant has been successfully used in studies of DGAT function wherein WT and mutated forms of plant DGAT1s have been expressed and thereby function or lack thereof in the plant inferred [28-32]. H1246MAT $\alpha$ yeast cells harboring an empty pYES2.1 vector plasmid or transformed with WT DGAT1 


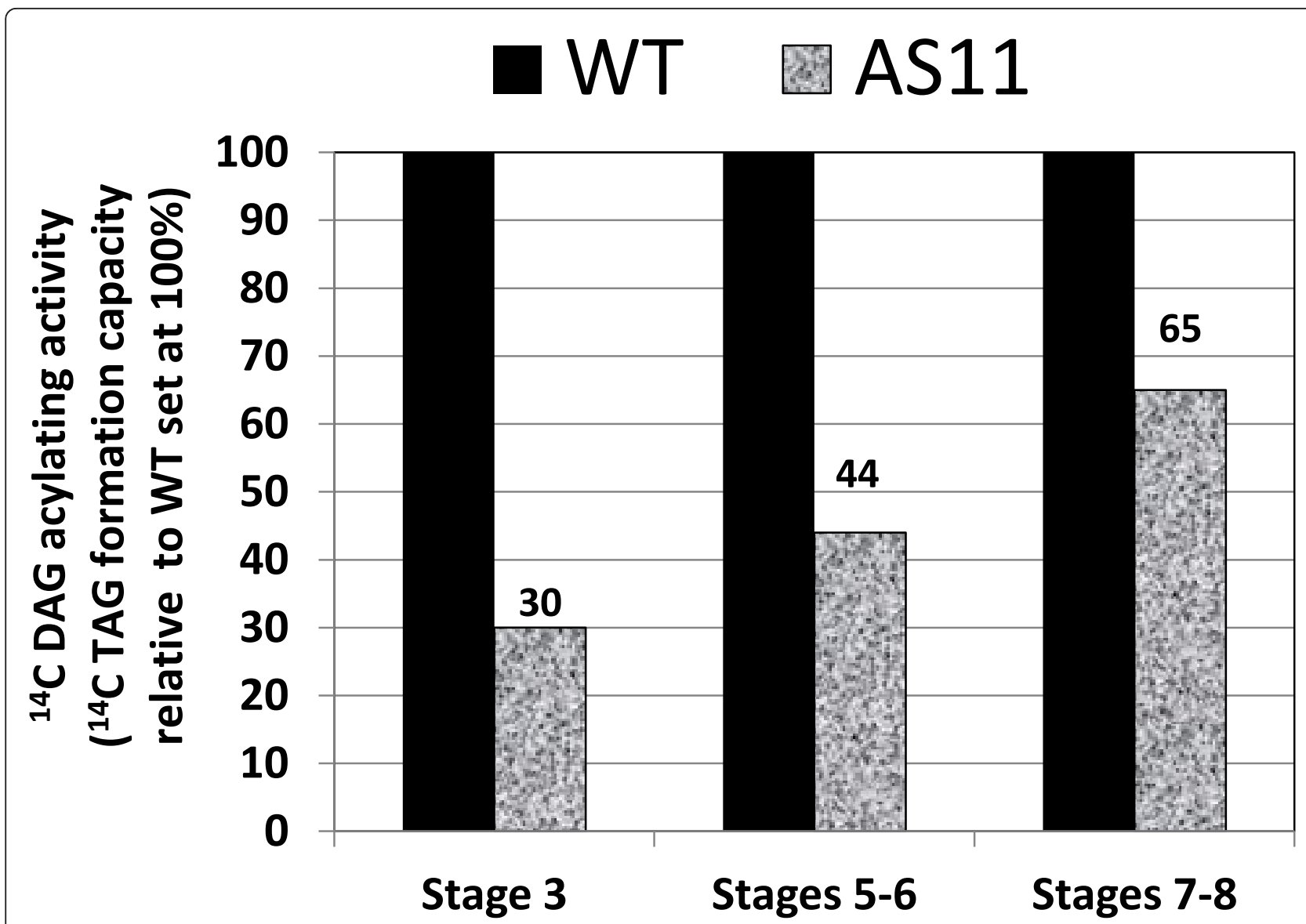

Figure 1 Comparison of protein fractions from mid-developing siliques containing WT and AS11 embryos at stages 3, 5-6 and 7-8 (as designated in "Methods") to incorporate ${ }^{14} \mathrm{C}-1,2$ diolein into ${ }^{14} \mathrm{C}$ TAG in the presence of 18:1-CoA.

cDNA were used as a negative and positive controls, respectively (Figure 2). A western blot of the microsomal membrane fractions isolated from the induced yeast H1246MAT $\alpha$ cells (Figure 2A) showed that both the mutated $A S 11$ and $A 7$ and WT DGAT1 proteins (calculated $\mathrm{M}_{\mathrm{r}}$ of $62.2 \mathrm{kD}, 53.9 \mathrm{kD}$ and $58.9 \mathrm{kD}$, respectively) were stably expressed. In the pYES plasmid-only transformant, there was neither detectable DGAT1 protein, nor non-specific cross-reactivity of the antibody with non-DGAT1 proteins of a similar size. The western showed that the AS11 DGAT1 was slightly larger (by 27 amino acids, probably due to the exon 2 repeat) than that of WT, while for the A7 DGAT1, the largest possible stable transcript (truncated at the start of T-DNA insertion) encoded a protein that was significantly smaller than WT. However, as shown in Figure 2B, the AS11 DGAT1 could not compensate for the inability to produce TAG in this yeast quadruple mutant. Equally, when we assayed the transformed yeast microsomal protein fractions in vitro for DGAT activity using unlabeled oleoyl-CoA (18:1) as an acyl donor, and ${ }^{14}$ C-sn-1,2 diolein (18:1) as acceptor, enzyme activity was not detected in the yeast strain harboring the mutated DGAT1 cDNA from the AS11 mutant and empty control pYES2.1 vector, but was found in the positive WT control. This indicated, perhaps not unexpectedly, but for the first time, that the mutated dgat 1 from $A S 11$ is nonfunctional. Equally, the truncated DGAT1 from the $A 7$ mutant (SALK-039456) was shown to be non-functional when expressed in H1246MAT $\alpha$ cells (Figure 2B).

Thus our results strongly support that DGAT1 is not functioning in the developing seed of the AS11 mutant line. Clearly, the (radiolabeled) TAG formation observed in protein fractions from AS11 was coming from another path and not via reduced DGAT1 catalysis.

Given the importance of PDAT1 in oil biosynthesis in Arabidopsis as we earlier defined [17], and combining this new information with the ${ }^{14} \mathrm{C}$ TAG biosynthesis results in assays of WT $v s$ AS11 protein fractions reported above, it raised the question that, if not from DGAT1, DGAT2 nor PDAT2, what biochemical steps besides PDAT1 may be critical for TAG biosynthesis? 


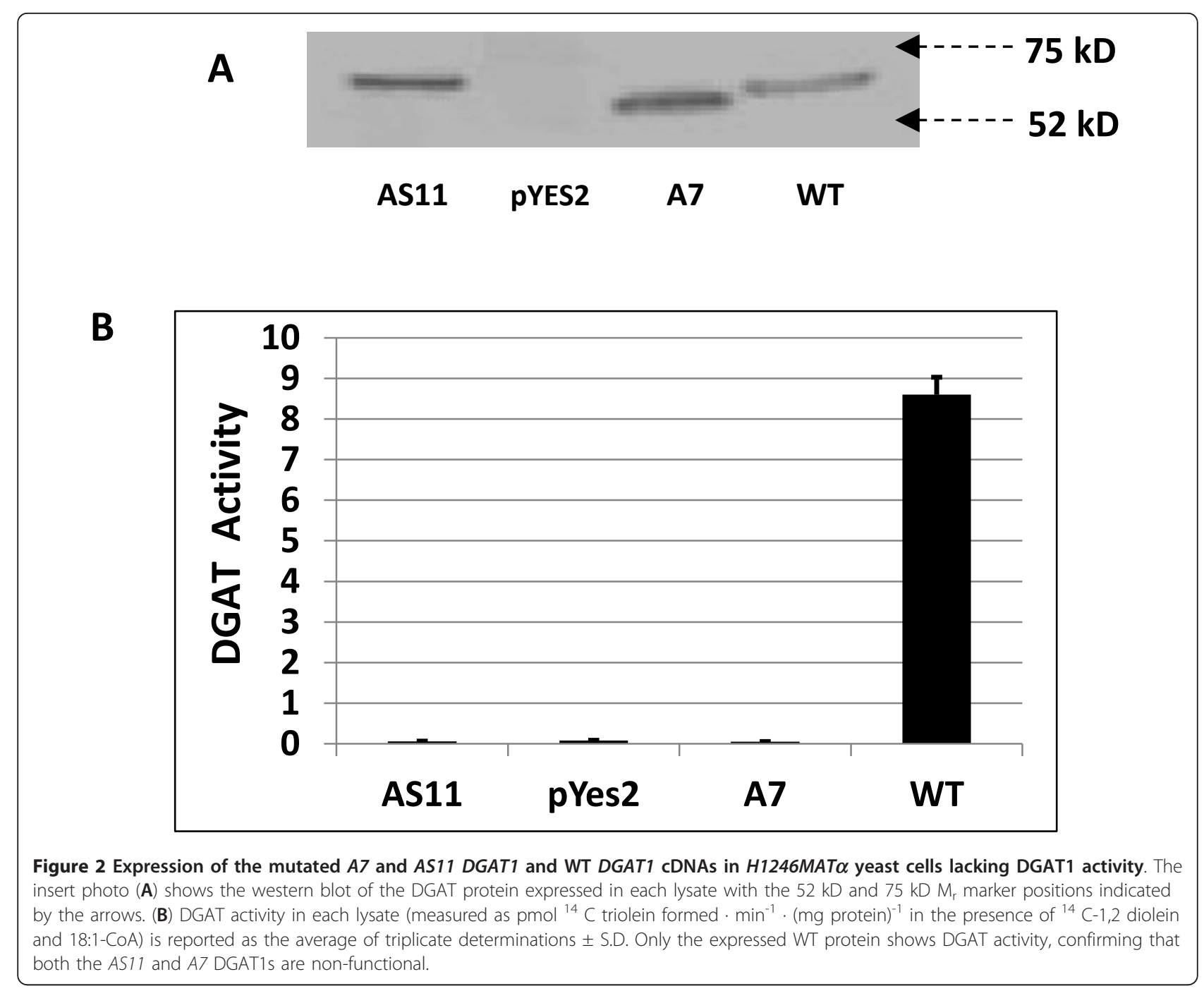

\section{AS11 microarray and qRT-PCR analyses}

These cumulative findings prompted us to examine a broader inventory of gene transcripts/encoded proteins that may be involved in regulating lipid biosynthesis in AS11 when DGAT1 activity is compromised, compared to their corresponding expression pattern in WT. Thus we performed a microarray analysis of gene expression in mid-developing seeds of mutant AS11 and WT Arabidopsis. Based on selected probable lipid assemblyrelated transcripts [33] differentially expressed through the microarray study, we complemented this with a semi-quantitative qRT-PCR analysis and compared the \% change in AS11 transcript relative to WT, for each. While neither method is truly quantitative, the qualitative trends in each study were highly consistent (Figure 3).

Some general observations from these combined gene expression studies follow: In $A S 11, F A E 1$ is down- regulated, FADs $2 \& 3$ are up-regulated; this is consistent with the AS11 acyl composition profile (reduced 20:1 and elevated 18:3). DGAT1 expression was not significantly affected. Interestingly, LPCAT2 was up-regulated by an average of $65 \%$, while $L P C A T 1$ expression was indifferent. PDAT2 was only marginally affected, but PDAT1 expression was up-regulated by an average of $62 \%$ compared to WT.

Given the critical role of PDAT1 in embryo development and TAG deposition in both pollen and seeds [17], we were interested in the relative differences in LPCAT1 and LPCAT2 expression between the AS11 mutant and WT. We hypothesized that an acyl-CoAdependent LPCAT may be critical to maintain the PC pool as one of the co-substrates for PDAT1-catalyzed TAG synthesis, particularly in the absence of a functional DGAT1, and performed a series of genetic and metabolic studies to examine these relationships. 


\section{घRT-PCR $\square$ Microarray}

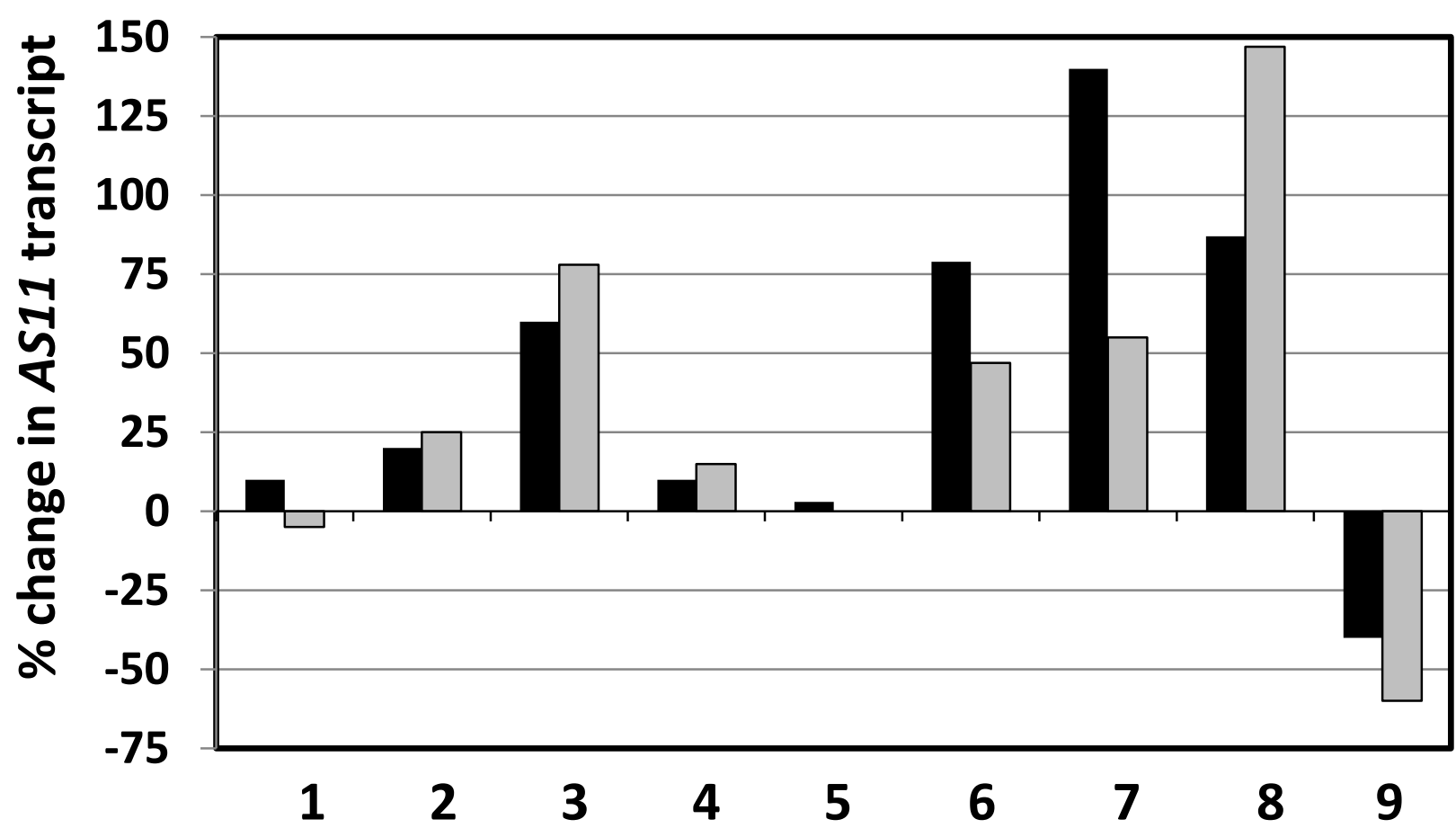

Figure 3 Comparison of change in expression of lipid metabolism genes selected from microarray data and from semi-quantitative RT-PCR data, each presented as \% change in AS11 transcript (relative to WT). Both assessments were conducted using the same triplicate biological RNA samples isolated from mid-developing siliques containing WT and AS11 embryos at stages 7-8. Genes are numbered 1-9 and named by their acronym and AGI number as follows: (1) DGAT1 (At2g19450); (2) DGAT2 (At3g51520); (3) PDAT1 (At5g13640); (4) PDAT2 (At3g44830); (5) LPCAT1 (At1g12640); (6) LPCAT2 (At1g63050); (7) FAD2 (At3g12120); (8) FAD3 (At2g29980); (9) FAE1 (At4g34250)

RT-PCR Characterization of the Ipcat1, Ipcat2 and dgat1 (AS11) mutants

In addition to the WT and AS11 lines, we selected accession lines with putative mutations in LPCAT1 and $L P C A T 2$, and characterized each of the individual mutants using RT-PCR to confirm the transcript phenotype of each line. As shown in Figure 4, in the putative lpcat1 mutant the ca $1100 \mathrm{bp}$ transcript was absent, but it was amplified in the control (WT) sample, indicating that the mutant At1g12640 is indeed a knockout for the LPCAT1 gene. Similarly as shown in Figure $5 \mathrm{~B}$, lane 2, the band amplified for the LPCAT2 transcript is absent in the putative lpcat 2 mutant, but present in the WT and AS11 mutant (lanes 1 and 3, respectively) confirming that line At1g63050 is a knockout for the LPCAT2 gene. As expected, both the WT and the lpcat 2 mutant samples are positive for the WT DGAT1 amplicon (Figure 5A, lanes 1 and 3, respectively). As shown in Figure 5A, lane 3, the AS11 mutant dgat1 transcript is present, encoding the inframe exon 2, 27-amino acid repeat, the amplified fragment being 81 bp larger than that found in the WT and in the lpcat 2 mutant. However, as demonstrated in Figure 2 and discussed above, though present, we have very strong evidence from yeast expression studies that the mutant dgat1 encoded by this transcript is non-functional.

\section{Metabolic studies}

We performed in vitro LPCAT assays in a time course incubation of protein fractions from $A S 11$ and WT middeveloping seed, in the presence of $s n-1$ palmitoyl- ${ }^{3} \mathrm{H}$ $\mathrm{LPC}+{ }^{14} \mathrm{C}-18: 1-\mathrm{CoA}$ and followed the ${ }^{3} \mathrm{H}$ and ${ }^{14} \mathrm{C}$ labeling patterns in PC. As shown in Figure 6A the ${ }^{3} \mathrm{H}$ accumulation pattern indicated that tritiated $\mathrm{PC}$ was rapidly synthesized from ${ }^{3} \mathrm{H} \mathrm{LPC}$ at a rate that was 3.5fold higher in AS11 than WT within 20 min. Based on the proportion of $s n-2{ }^{14} \mathrm{C}$ oleoyl moieties incorporated into PC, the LPCAT activity was consistently 40-60\% higher in $A S 11$ at all time points (Figure 6B). Equally, the proportion of ${ }^{3} \mathrm{H}$ in LPC concomitantly decreased in AS11 relative to WT over this period (data not shown). This indicated that in AS11, the LPCAT activity was strongly enhanced relative to WT. 


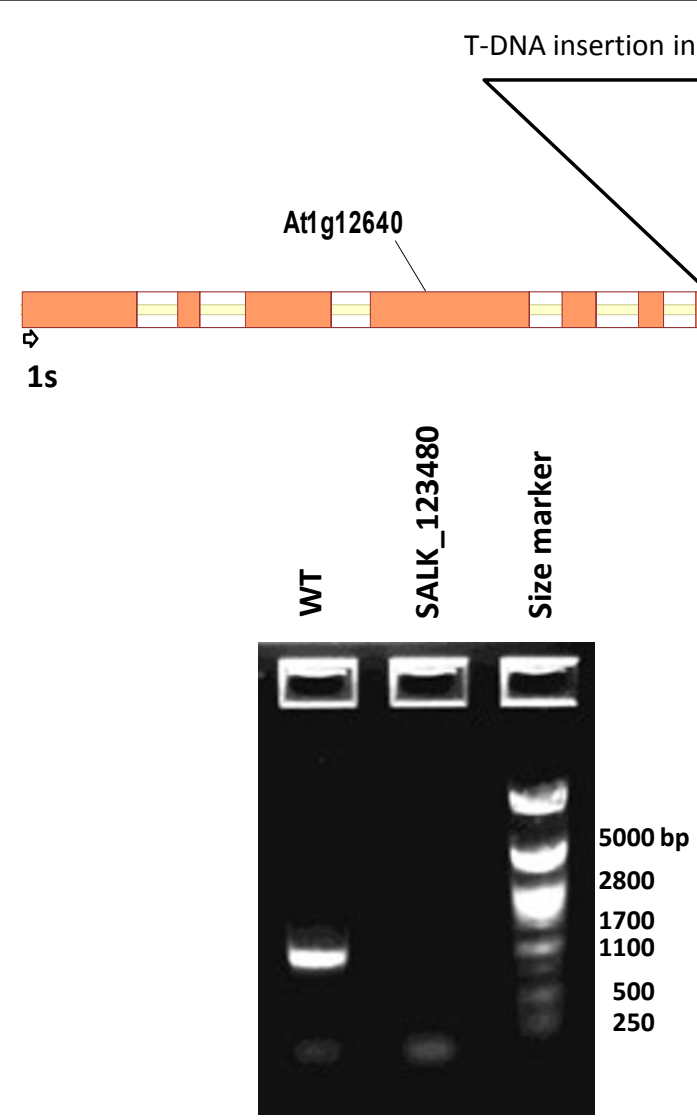

Figure 4 LPCAT1 expression in Col WT and Ipcat1 T-DNA insertion knockout mutant Salk_123480. RT-PCR was conducted as described in "Methods" and show that the transcript for LPCAT1 is absent in the Salk_123480 mutant. The map shows the position of the T-DNA insertion in LPCAT1 gene.

We also performed an in vivo feeding study wherein we supplied ${ }^{14} \mathrm{C}$ acetate to bolted stems containing pods with mid-developing seeds of both AS11 and WT plants, and then analyzed the label patterns in various lipid fractions in the mid-developing seeds after a chase period of 7, 24 and $30 \mathrm{~h}$. Two major differences were immediately obvious (Figure 7): $A S 11$ showed a higher relative incorporation of ${ }^{14} \mathrm{C}$ into $\mathrm{PC}$ and a lower relative incorporation of ${ }^{14} \mathrm{C}$ into TAG over the time course. These trends were entirely consistent with an elevated LPCAT activity and reduced TAG synthesis in AS11 as shown in the in vitro LPCAT (Figure 6) and DGAT1 (Figure 1) assays.

Based on these trends we were confident that in addition to DGAT1 and PDAT1, LPCAT plays a significant role in TAG biosynthesis, and postulated that it is important not only for membrane development and acyl turnover therein, but also to replenish the supply of PC for PDAT1-catalyzed TAG biosynthesis in the developing seed. To resolve this question we needed to study what occurs when both DGAT1 and LPCAT expression are co-disrupted.

\section{Genetic crosses}

It had previously been shown, following expression in a yeast lca1 $1 \Delta$ mutant background, that both Arabidopsis LPCAT1 (At1g12640) and LPCAT2 (At1g63050) are functional LPCATs. In the presence of $s n-1\left[{ }^{14} \mathrm{C}\right] 16: 0$ LPC and 18:1-CoA (the same combination of substrates used in the current study), expressed LPCAT1 and LPCAT2 proteins had activities of $12 \mathrm{nmol} / \mathrm{min} / \mathrm{mg} / \mathrm{mg}$ protein and $18 \mathrm{nmol} / \mathrm{min} / \mathrm{mg}$ protein, respectively [34].

As confirmed by RT-PCR (discussed above), lpact 1 (At1g12640) and lpcat2 (At1g63050) are T-DNA insertion knockouts for their respective genes, while AS11 encodes a mutated and non-functional (Figure 2) dgat1. To examine the relationship between these two LPCATs and DGAT1 in TAG biosynthesis, we performed crosses of the AS11 dgat1 with the lpcat1 or the lpcat2 T-DNA insertion knock-out mutants and characterized the hemizygous/homozygous and double knockout seed oil profiles.

dgat $1 \times$ Ipcat 1

The lpcat1 mutant is devoid of any significant oil phenotype compared to its null segregant or to WT (Figure 


\section{$\begin{array}{lllll}1 & 2 & 3 & 4 & 5\end{array}$}

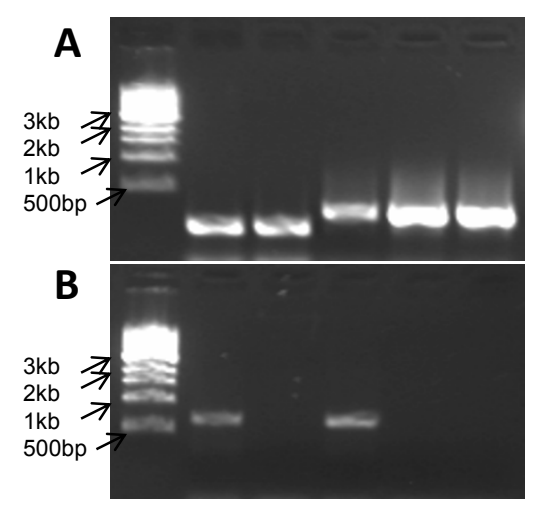

AS11(At2g19450)

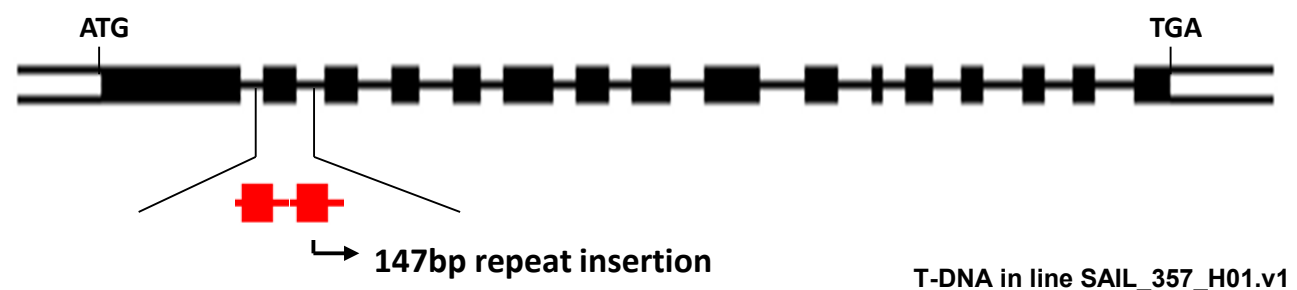

LPCAT2(At1g63050)

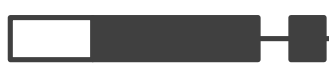

Figure 5 (A) DGAT1 expression Lane 1: WT; Lane 2 Ipcat2 H single mutant, Lane 3-AS11 dgat1 single mutant; Lane 4 dgat1/lpcat2 H/H double mutant line \#6-3-10-19; Lane 5 dgat1/lpcat2 H/H double mutant line \#6-3-20-1. (B) LPCAT2 expression: Lane 1: WT; Lane 2 lpcat2 H single mutant SAlL_357_H01, Lane 3-AS11 dgat1 single mutant; Lane 4 dgat1/lpcat2 H/H double mutant line \#6-3-10-19; Lane 5 dgat1//pcat2 H/H double mutant line \#6-3-20-1. RT-PCR was conducted using DGAT1-specific primers (A) and LPCAT2-specific primers (B) as described in

"Methods". The maps show the position of 147 bp repeat insertion in the AS11 DGAT1 gene and the T-DNA insertion site in the LPCAT2 gene.

8). Crosses of AS11 (dgat1) with the lpcat1 yielded progeny homozygous for both mutations. The double homozygous mutant showed normal plant development and seed set. Zhang et al. [17] showed that without DGAT1, the PDAT1 route contributed $75 \%$ oil in AS11; thus even without combined contributions from [DGAT1 + LPCAT1], the PDAT1 route could still provide up to $70 \%$ oil in the developing AS11 seeds. In other words, the co-incident loss of LPCAT1 with DGAT1 reduced the capacity for PDAT1-catalyzed oil synthesis by only $5 \%$.

While oil content in the dgat1/lpcat1 $\mathrm{H} / \mathrm{H}$ line is reduced, it is interesting to note that with respect to seed weight, there is a significant increase (by ca 6\%) relative to the dgat1/lpcat1 null/null control (Figure 9). Thus, while there was no penalty in this regard, further study is needed to ascertain which components of the seed e.g. protein or cellulosic biomass of the seed coat) account for this observation.
The fatty acid profile from the lpcat1 mutant is identical to that of its null segregant (Figure 10). The AS11 (dgat1) x lpcat1 double knockout lines show an AS11like profile (low 18:1 and 20:1; high 18:3); the 18:3 proportion is about $2-5 \%$ lower than in $A S 11$ alone. The latter is not inconsistent with the fact that LPCATs are involved in the shuttling of $18: 1$ moieties to the PC backbone for desaturation by FADs 2 and 3 .

dgat $1 \times$ Ipcat 2

The lpcat 2 mutant does not have a significant oil phenotype compared to its null segregant-i.e. it is similar to WT (Figure 11). The fatty acid composition is essentially identical to WT and to that exhibited by the LPCAT2 null segregant (Figure 12). When we performed the AS11(dgat1) x lpcat2 crosses, the dgat1/lpcat $2 \mathrm{He} / \mathrm{H}$ shows only a small reduction in oil content which suggests that when DGAT1 is partially expressed $(\mathrm{He})$, and LPCAT2 is knocked out $(\mathrm{H})$, there is a reduced capacity for compensation in TAG biosynthesis, probably 

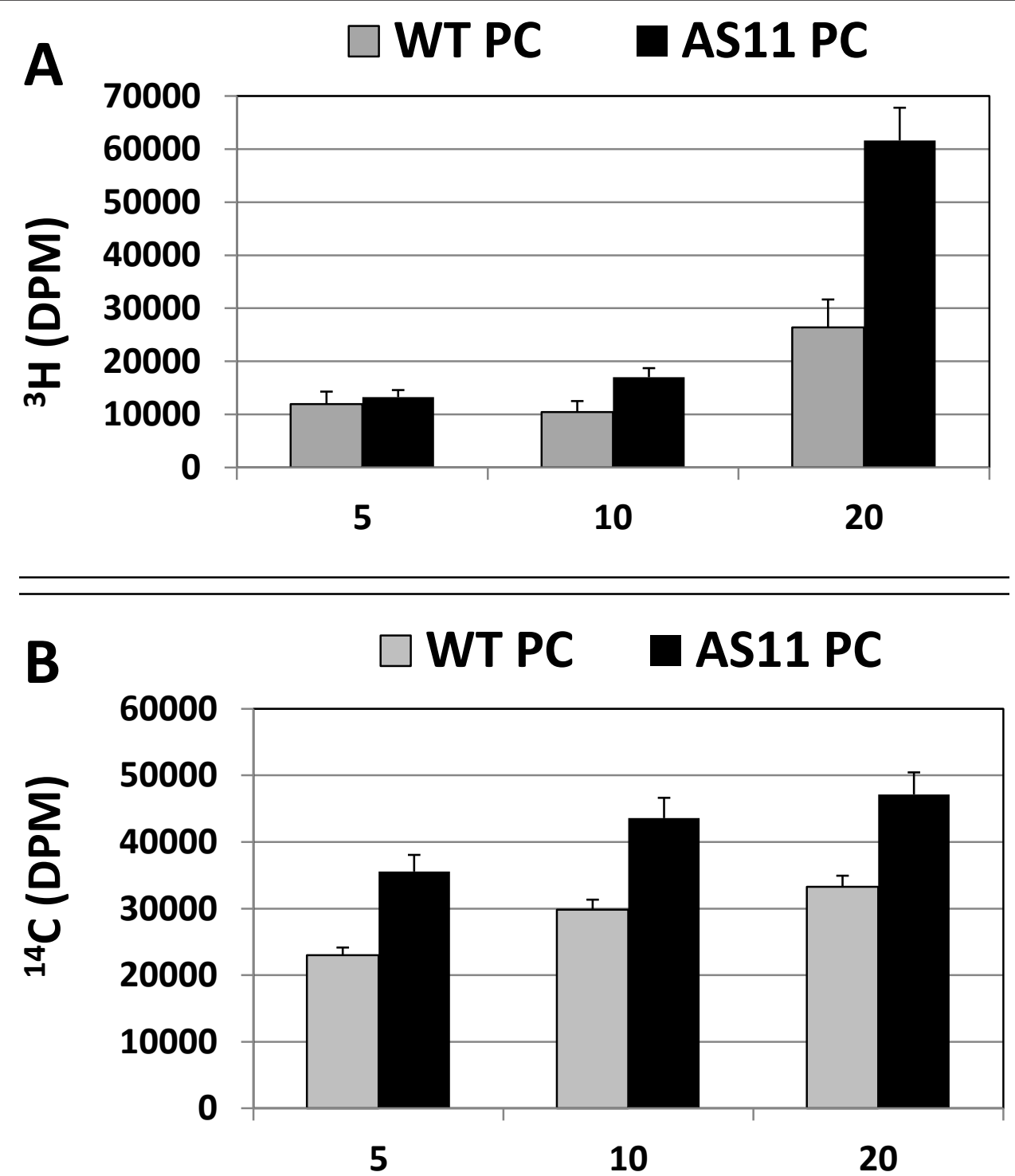

Figure 6 Relative LPCAT activity in protein fractions from mid-developing siliques containing WT and AS11 embryos at stages 7-8 (as designated in Methods"). LPCAT activity was monitored by appearance of (A) ${ }^{3} \mathrm{H}$ and (B) ${ }^{14} \mathrm{C}$ label in PC, during a 20 min incubation of WT or AS11 protein in the presence of L- $\alpha$-palmitoyl- $\left[1-{ }^{3} \mathrm{H}\right.$ methyl] lyso-3-phosphatidylcholine and $\left[1-{ }^{14} \mathrm{C}\right] 18: 1-\mathrm{Co}$. The data represent the average of two experimental replicates \pm S.D. While absolute values differed, similar trends were observed in a second independent biological replication.

because PDAT is insufficiently "fueled" with its co-substrate, PC. In these lines there is a severe penalty on seed development; there are many gaps in developing siliques due to non-fertilized ovules (Additional file 1: Figure S2A,B).

Relative to the dgat1/lpcat2 double null, in the lpcat2 $\mathrm{H}$ and dgat1/lpcat $2 \mathrm{He} / \mathrm{H}$ lines, seed weight is increased by about $10 \%$ (Figure 13). However, the dgat $1 /$ lpcat $2 \mathrm{H} /$ $\mathrm{H}$ lines show an strong decrease in seed weight even beyond that observed in the dgat1 mutant alone or when the double mutant genotype has dgat 1 in the homozygous condition, Taken together with the seed weight increases cited above for the lpcat1 mutant studies, these findings make it very clear that both the lpcat 1 or lpcat 2 mutations have a complicated effect on seed weight which may or may not be related to changes in oil content.

The acyl composition of the dgat1/lpcat $2 \mathrm{He} / \mathrm{H}$ lines was again essentially like the LPCAT2 NS (Figure 12). In contrast, the dgat1/lpcat $2 \mathrm{H} / \mathrm{He}$ lines have an AS11-like reduction in oil content which is about $70 \%$ of that found in the WT and the LPCAT2 null segregant (Figure 11). Interestingly, there is no developmental penalty in this heterozygous combination and seed set is normal 


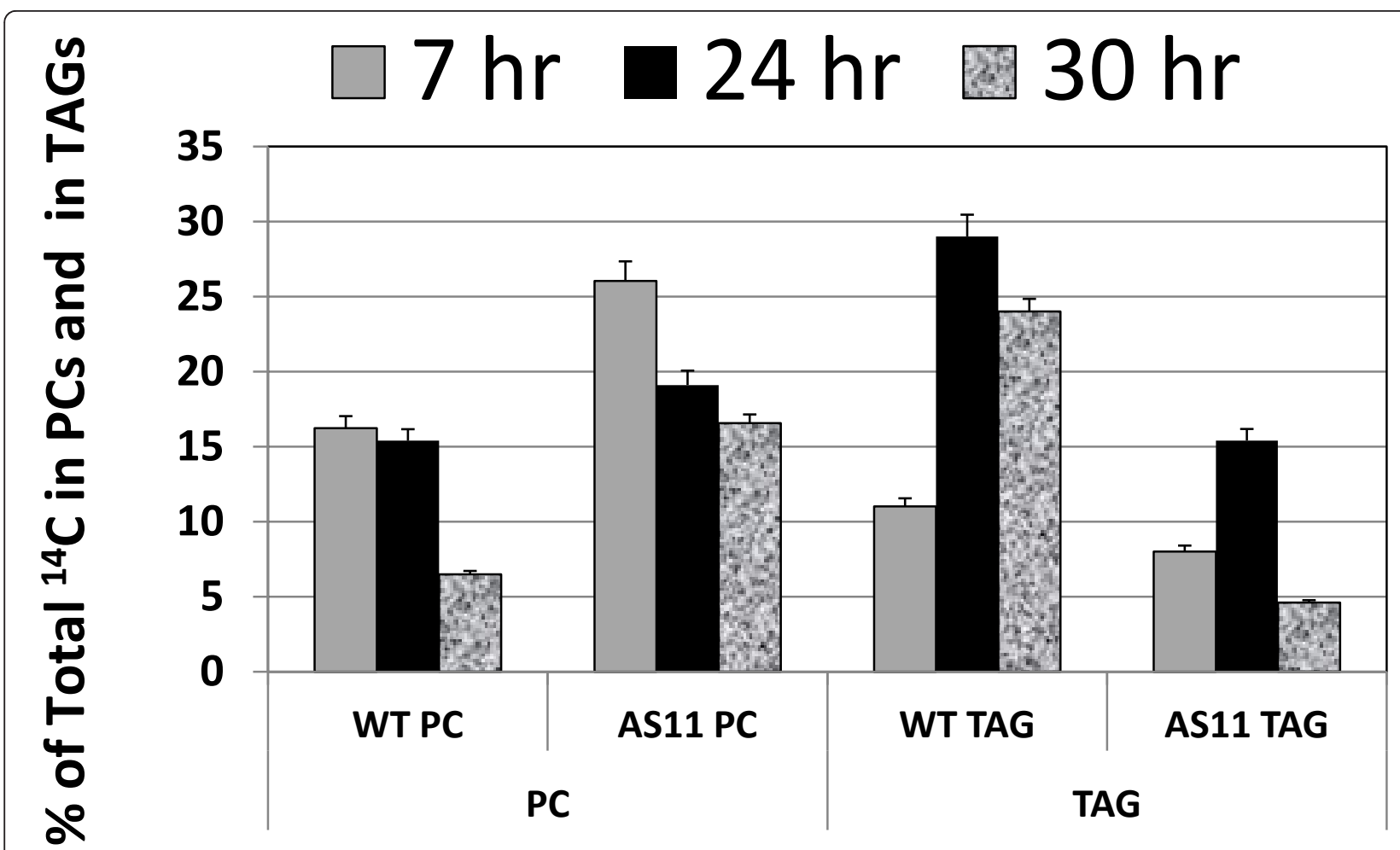

Figure 7 Relative incorporation of ${ }^{14} \mathrm{C}$ into PC and into TAG in WT vs AS11 following an in vivo feeding experiment in which severed bolted stems containing mid-developing siliques were supplied with $1{ }^{14} \mathrm{C}$ sodium acetate over a $30 \mathrm{~h}$ time course. Seeds were harvested at each time point and seed lipids isolated and quantified as described in Methods. Results are the average of triplicate experimental determinations \pm S.D

as in the AS11 background, provided expression of LPCAT2 is in the heterozygous state (Additional file 1: Figure S3). Not unexpectedly, the acyl composition of the oil in these lines is consistent with the AS11 profile (Figure 12).

The results were quite different in the AS11 (dgat1) x lpcat 2 double homozygous mutant. Shown in Figure 14 is a PCR confirmation of the genotype of putative $\mathrm{H} / \mathrm{H}$ lines \# 6-3-10-19, \# 6-3-20-1 compared to WT, AS11 and lpcat2 lines. AS11, and dgat1/lpcat2 H/H lines \# 63-10-19 and \# 6-3-20-1 have the expected 147 bp insert indicating they are homozygous for the insertion mutation resulting in a non-functional DGAT1 (as shown in Figure 2, above), while the WT and lpcat2 samples do not. T-DNA screening was done on the samples of the same lines to test for the presence of LPCAT2. Samples were amplified using [left primer + right primer] or with [right primer + left border 2] primer. The lpcat2 and the dgat1/lpcat2 $\mathrm{H} / \mathrm{H}$ lines \# 6-3-10-19 and \# 6-3-20-1 have a homozygous knockout of the LPCAT2 gene. qRT-PCR also confirmed the transcript profile in these two lines for both dgat1 (Figure 5A, lanes 4 and 5) and lpcat2, (Figure 5B, lanes 4 and 5).). Biochemically, in the double homozygous mutant (dgat1/lpcat $2 \mathrm{H} / \mathrm{H})$ there is a severe penalty on TAG synthesis; there is a $65 \%$ reduction in oil content relative to WT: Mature seeds have only about $30 \%$ oil content relative to the WT and the LPCAT2 null segregant proportions (Figure 11). Without DGAT1 (AS11), recent studies suggested that PDAT1 catalyzes the synthesis of essentially all the oil which accumulates at $70-75 \%$ of that found in WT [17]. Without both LPCAT2 and DGAT1, PDAT1 is able to synthesize only about $35 \%$ of WT oil levels. Thus, the loss of LPCAT2 strongly reduces the capacity of PDAT1 to synthesize oil. This suggests a strong link between PDAT1 and its supply of PC as a co-substrate in TAG synthesis; clearly LPCAT2 plays a strong role in supplying this PC. When the DGAT1 mutation is homozygous, the AS11 acyl profile dominates when the LPCAT2 mutation is in the He condition (Figure 12). However, in both the lpcat 2 homozygous mutant and in the double mutant dgat1/lpcat $2 \mathrm{H} / \mathrm{H}$ lines, the $20: 1$ and 18:1 are slightly increased while 18:2 is decreased (Figure 12). It is significant to note that Shen et al. [34] demonstrated that an Arabisdopsis line transformed with an LPCAT1-LPCAT2 double RNAi construct, partially repressing the expression of both genes, also exhibited a $3 \%$ increase in the proportion of $20: 1$, a $1.5 \%$ increase in 


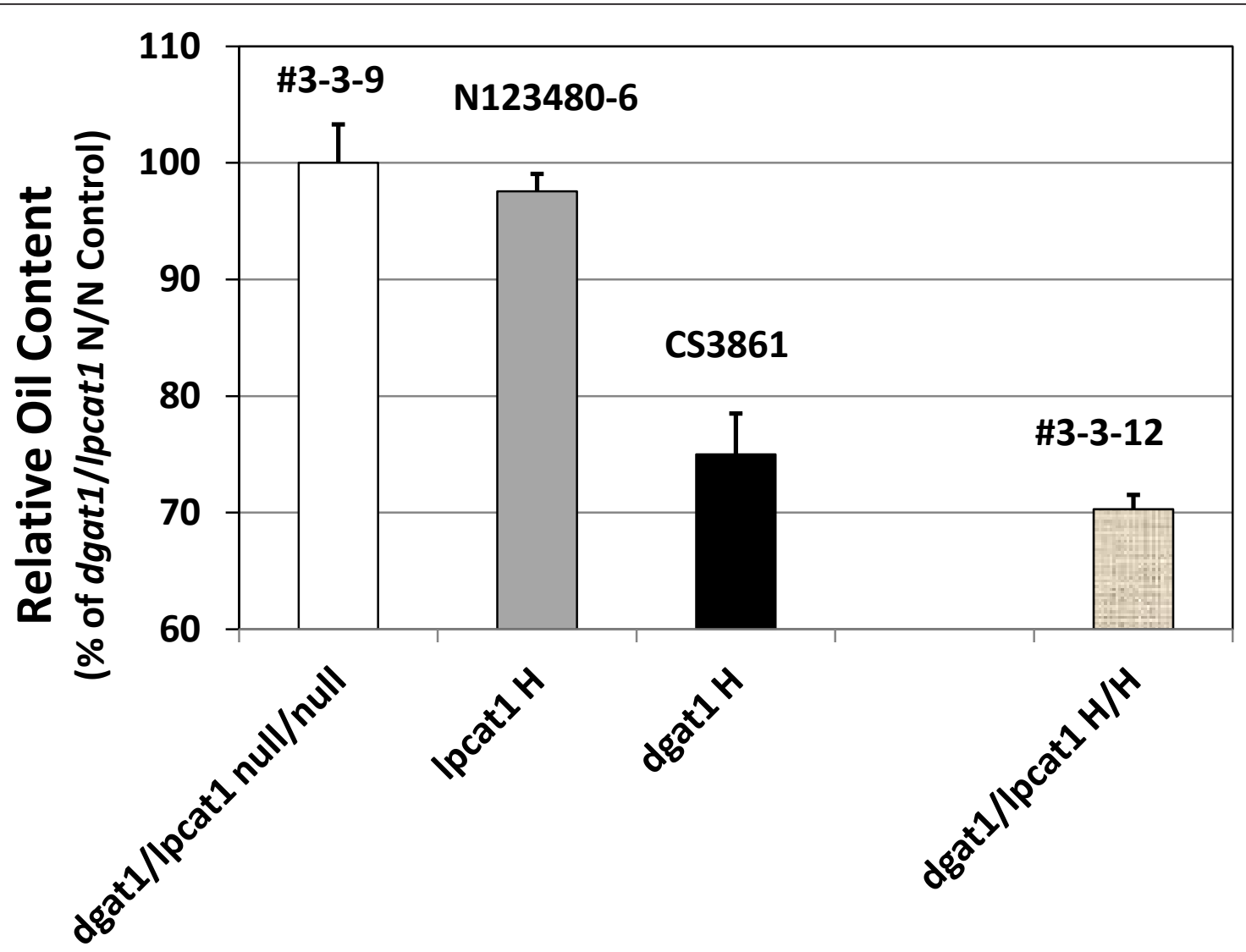

Figure 8 Relative oil content in $\mathbf{F}_{2}$ seeds from crosses of Ipcat1 and AS11 mutants. Line numbers are designated as listed in Additional file 2: Table S3, with genotypes designated on the $\times$ axis. dgat1/lpcat1 null for both mutations \# 3-3-9; Ipcat1 homozygous mutant N123480-6; AS11 dgat1 homozygous mutant CS3861; dgat1/Ipcat1 homozygous for both mutations \#3-3-12. The values are reported relative to the double null controls set at $100 \%$. Values are the average of triplicate determinations \pm S.D.

18:1 and a concomitant $3 \%$ decrease in $18: 2$ in seed TAGs compared to WT. While the current phenotype is difficult to interpret without further study, these findings will be important in future analyses of LPCATs in terms of substrate selectivity and potential channeling of fatty acids into oil.

Plant growth and development is also delayed in the double homozygous mutant (dgat1/lpcat $2 \mathrm{H} / \mathrm{H})$, as it is in AS11: the seedlings are small and slow to develop (Figure 15A). Once transferred to soil, bolting to enter the generative phase is delayed in dgat1/lpcat $2 \mathrm{H} / \mathrm{H}$ (Figure 15B), but is normal in dgat1/lpcat2 H/He (Figure $15 \mathrm{C}$ ). Seed development is poor, as in the dgat1/ lpcat $2 \mathrm{He} / \mathrm{H}$ lines; there are many non-fertilized ovules in developing siliques resulting in seed which is visibly reduced compared to that normally observed in WT or LPCAT2 null segregant (NS). Thus, it is clear that some level of LPCAT2 expression is necessary for normal seed development, especially in an AS11 background. However, future investigations of this preliminary observation, including scoring of developmental phenotypes and a microscopic study of pollen viability, will be required to confirm a disturbed pollen functionality as a result of the double mutation in the dgat1/lpcat2 $\mathrm{H} / \mathrm{H}$ lines.

Summarizing the mutant crossing experiments, in lpcat $2 \times$ dgat1, oil synthesis, vegetative growth and seed set are severely penalized. Compared to the relative oil content in dgat 1 alone ( $75 \%$ of WT), there is only a $5 \%$ further relative decrease in dgat1/lpcat1 oil (70\% of WT) which suggests that LPCAT2 is the major determinant and can almost fully compensate for the loss of LPCAT1. In contrast, in the co-incident absence of LPCAT2 and DGAT1 (lpcat2 $\times$ dgat1), relative oil content drops to $30 \%$ of WT, which suggests that LPCAT1 can only partially compensate for the loss of LPCAT2 with respect to oil. Metabolic and microarray studies in AS11 alone support this hypothesis as LPCAT2 expression and LPCAT activity are up-regulated in the absence of DGAT1. In addition, the acyl profiles indicate that any altered oil composition phenotype observed in the lpcat $2 \times$ dgat 1 is predominantly influenced by the dgat 1 lesion. Collectively, the data from the crosses of AS11 


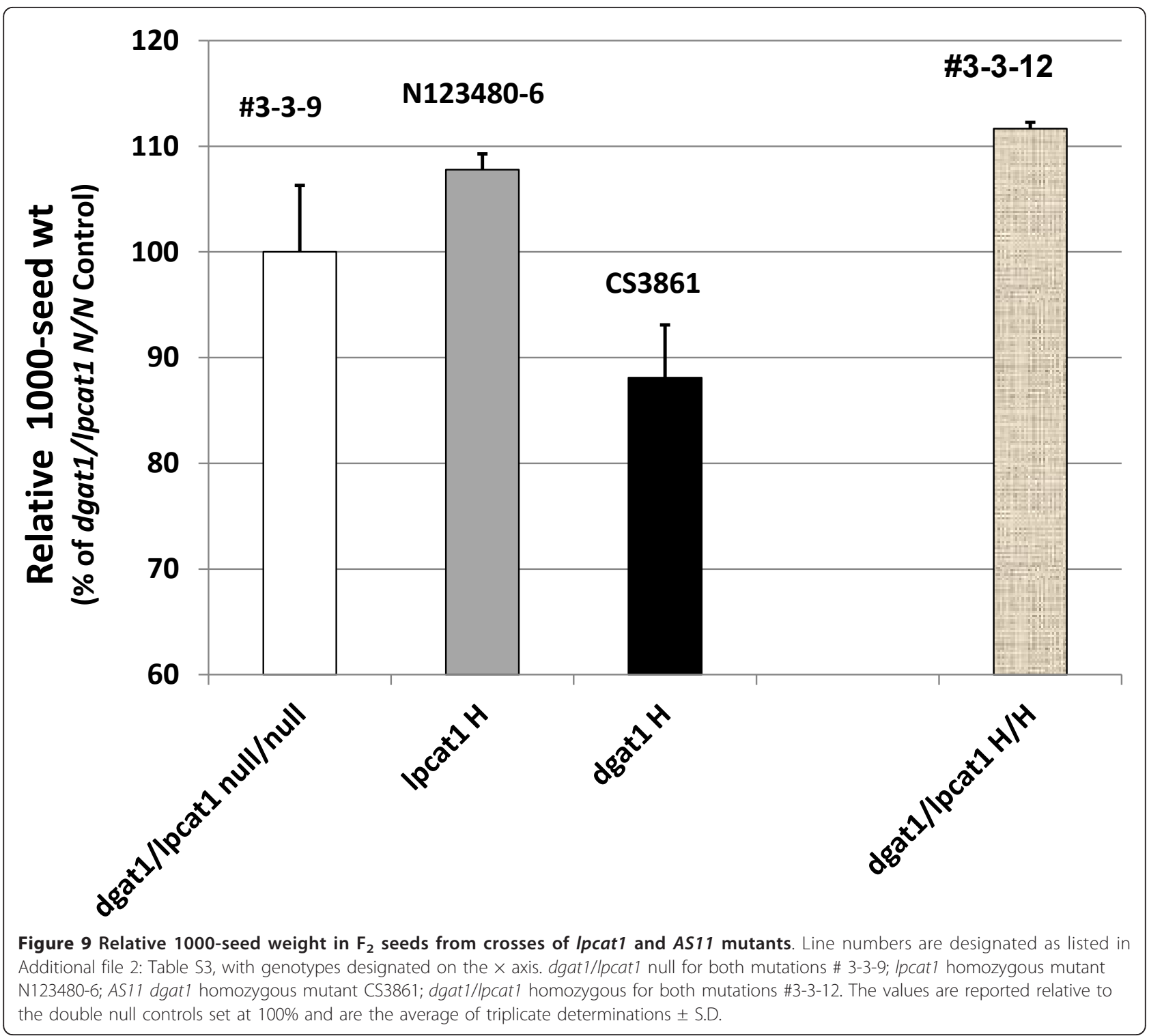

with lpcat 1 or lpcat 2 mutants strongly suggest that in an AS11 background, while both LPCAT2 and LPCAT1 can contribute to PDAT1-catalyzed TAG synthesis, it is LPCAT2 up-regulation (and not LPCAT1), which is primarily responsible for assisting in PDAT1-catalyzed TAG biosynthesis by supplying an enhanced pool of PC as co-substrate to transfer $s n-2$ moieties to the $s n-3$ position of the enlarged AS11 DAG pool. Nonetheless, while LPCAT2 is the most important for maintaining PDAT1-catalyzed TAG synthesis in the AS11 background, the partial over-lapping function of LPCATs 1 and 2 identified in this study require further investigation to determine their relative roles in oil synthesis when DGAT1 is functional in a WT background.

The recent discovery of a phosphatidylcholine: diacylglycerol cholinephosphotransferase (PDCT), encoded by the Arabidopsis ROD1 gene, indicates that the issue of DAG-PC interconversion during TAG biosynthesis is considerably more complex than previously thought [35]. The PDCT enzyme catalyzes transfer of the phosphocholine headgroup from PC to DAG and is thought to be a major reaction for the transfer of $18: 1$ into PC for desaturation by FAD2 and FAD3, as well as enabling the reverse transfer of 18:2 and 18:3 into the TAG synthesis pathway. In the rod 1 mutant, the accumulation of PUFAs in TAGs is reduced by $40 \%$. However, in AS11 where 18:3 is strongly increased, and notably, shows a strong stereospecific shift to the $s n-3$ position of TAGs [6], it is unclear whether there is a strong involvement of PDCT; were it so, one would expect a more even distribution of $18: 3$ at the $s n-2$ and $s n-3$ positions. However, the strong re-distribution of $18: 3$ 


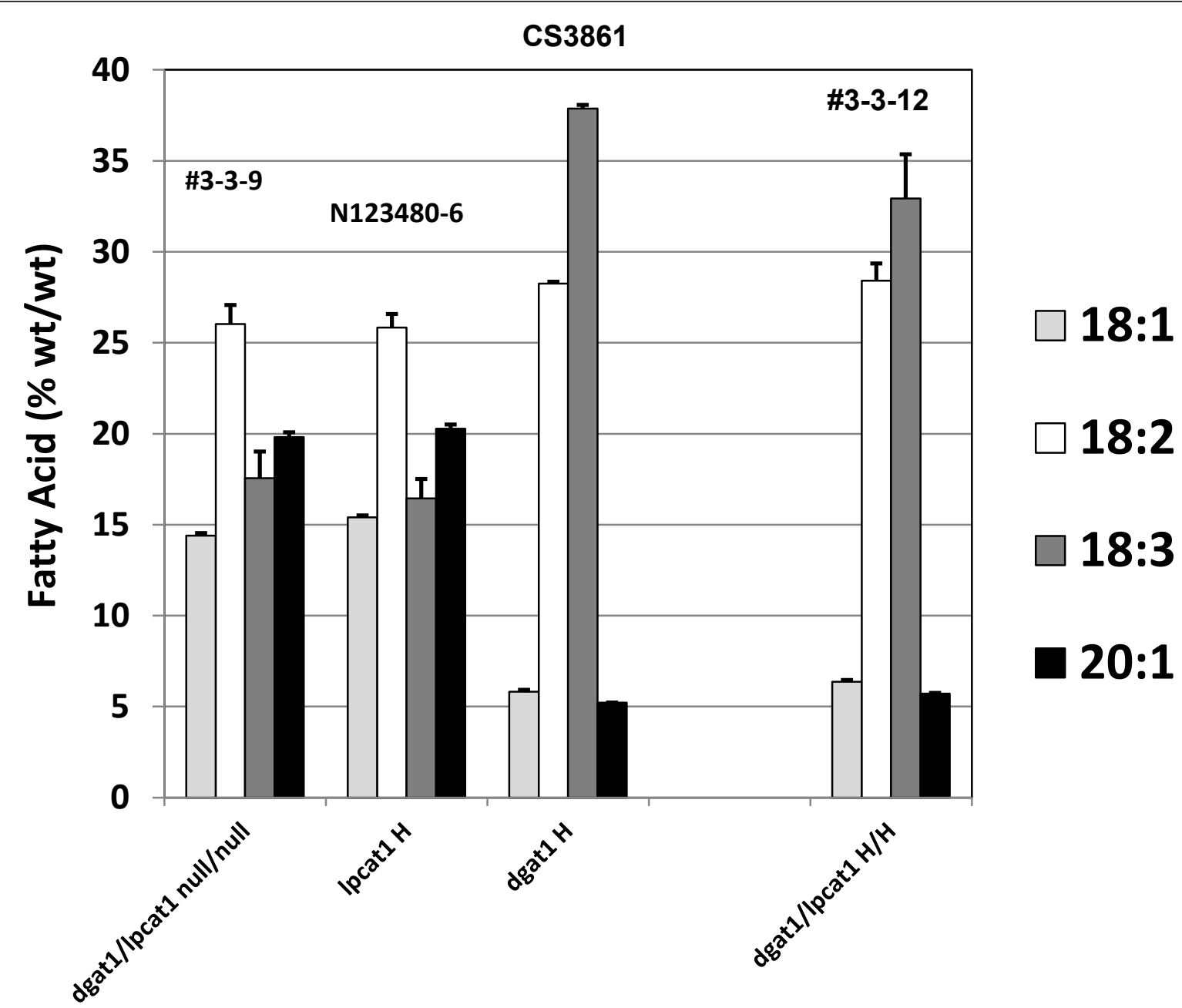

Figure 10 Compositional changes in key fatty acids in TAGs from $\mathrm{F}_{2}$ seeds of crosses of Ipcat 1 and AS11 mutants. Line numbers are designated as listed in Additional file 2: Table S3, with genotypes designated on the $\times$ axis. dgat1/lpcat1 null for both mutations \# 3-3-9; Ipcat1 homozygous mutant N123480-6; AS11 dgat1 homozygous mutant CS3861; dgat1/lpcat1 homozygous for both mutations \#3-3-12. The values are reported as \% (wt/wt) and are the average of triplicate determinations \pm S.D.

proportions to the $s n-3$ and away from the $s n-2$ position in AS11 suggests that the major shift in positional composition has to do with PDAT1 catalysis. Thus there are still many questions to address with respect to the roles ROD1 and other PC-related metabolic steps such as CPTase might play in oil synthesis in Arabidopsis and in AS11 in particular.

\section{Conclusions}

Based on the cumulative results of these studies, in Figure 16 we summarize our current hypothesis regarding the TAG biosynthesis pathway in WT $v s$. that observed when DGAT1 is compromised as in AS11. When both DGAT1 and PDAT1 are operating they both contribute to TAG synthesis, but the consensus of recent literature supports PDAT1 as a major route in Arabidopsis. When DGAT1 is eliminated, the PDAT1 pathway produces up to $75 \%$ of WT oil levels [17]. PDAT1 acylates the $s n-3$ position of DAGs which are accumulating (in the absence of DGAT1), to give TAG. The acyl composition of AS11 with highly enhanced PUFAs and reduced VLCFAs at the $s n-3$ position [6] supports the donation of acyl groups, especially 18:3, from the $s n$ - 2 position of $\mathrm{PC}$ to the $s n-3$ position of DAG as the major route for TAG synthesis in Arabidopsis.

This adjustment in the TAG assembly route in $A S 11$ is aided by enhanced LPCAT2 activity to supply the additional PC required by PDAT1. Given these findings and the implicit plasticity of Arabidopsis TAG assembly mechanisms to overcome critical bottlenecks, it will be interesting to determine the relative roles played by DGAT1 and [PDAT1 + LPCAT2] during TAG assembly in higher oilseeds (e.g. canola, soybean, sunflower, safflower, flax). 


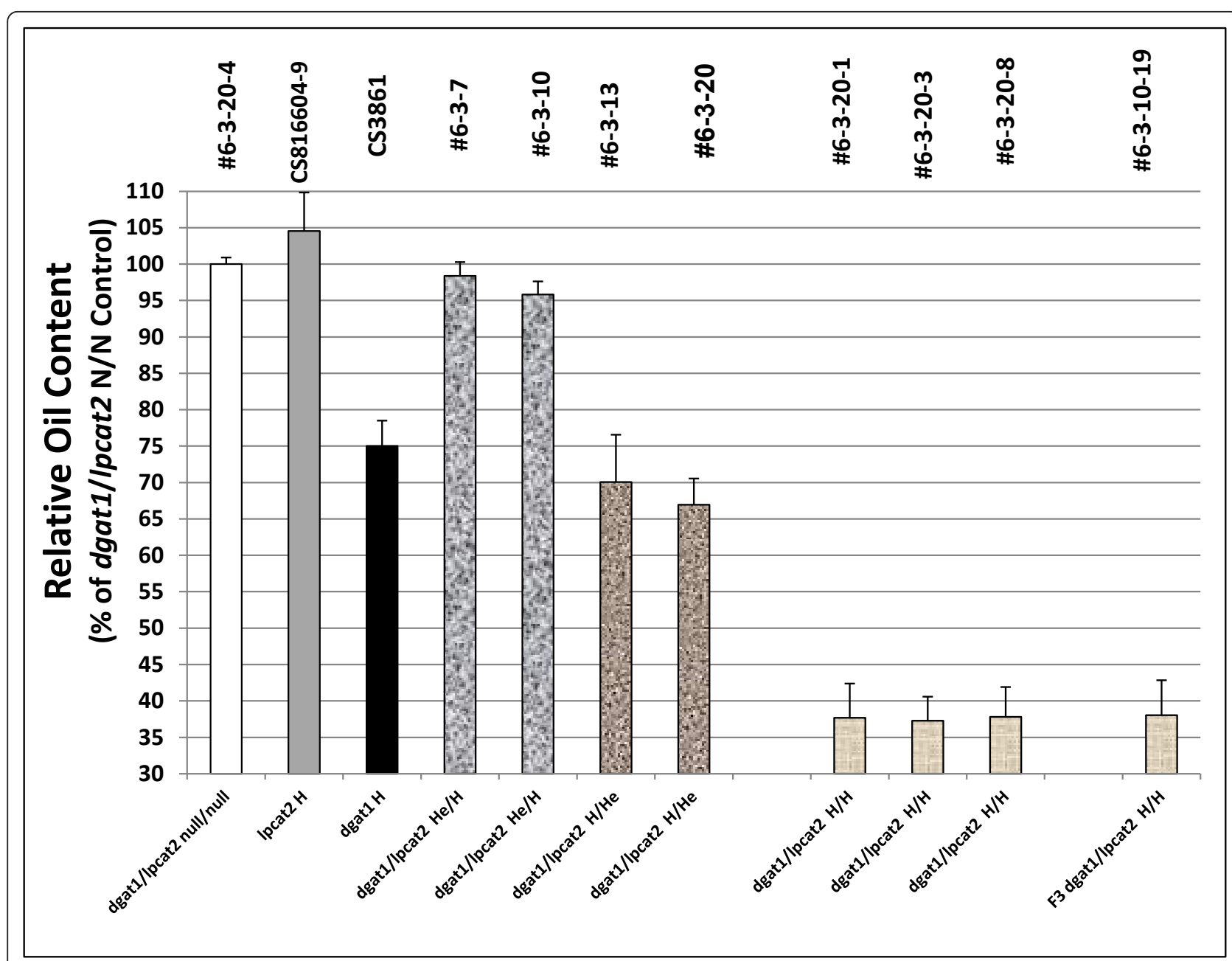

Figure 11 Relative oil content in $\mathbf{F}_{2}$ seeds from crosses of Ipcat2 and AS11 mutants. Line numbers are designated as listed in Additional file 2: Table S3, with genotypes designated on the $\times$ axis. dgat1/lpcat2 null for both mutations \# 6-3-20-4; Ipcat2 homozygous mutant CS8166049; AS11 dgat1 homozygous mutant CS3861; dgat1/lpcat2 He/H \#6-3-7 and \# 6-3-10; dgat1/lpcat2 H/He \# 6-3-13 and \# 6-3-20; dgat1/lpcat2 homozygous for both mutations \#6-3-20-1, \# 6-3-20-3 and \# 6-3-20-8; $F_{3}$ dgat1/lpcat2 homozygous for both mutations \# 6-3-10-19. The values are reported relative to the double null controls set at $100 \%$ and are the average of triplicate determinations \pm S.D.

\section{Methods}

\section{Plant materials and growth conditions}

Arabidopsis lines, WT (ecotype Columbia-0), and AS11 (CS3861) EMS-induced mutant, and A7 (SALK_039456), lpcat1 (SALK_123480) and lpcat2 (SAIL_357_H01) TDNA insertion mutant lines (all in a Columbia background) were obtained from the Salk Institute via the Nottingham Arabidopsis Stock Centre (University of Nottingham, UK). Seeds of these lines and progeny from genetic crosses were grown in a growth chamber at $22^{\circ} \mathrm{C}$ with photoperiod of $16 \mathrm{~h}$ light $\left(120 \mu \mathrm{E} \cdot \mathrm{m}^{-2} \cdot \mathrm{s}^{-1}\right)$ and $8 \mathrm{~h}$ dark.

Arabidopsis siliques containing mid-green developing embryos (pooled siliques at stages 6- 8, as designated on the University of Toronto Arabidopsis eFP Expression
Browser at: http://bar.utoronto.ca/affydb/cgi-bin/affy_ db_exprss_browser_in.cgi; see e.g. seed staging in LPCAT2 developmental map at http://bbc.botany.utoronto.ca/efp/cgi-bin/efpWeb.cgi?primaryGene=AT1G63050\&modeInput=Absolute), were harvested and frozen at $-80^{\circ} \mathrm{C}$ for lipid analyses, enzyme assays and DNA and RNA extraction.

\section{Lipid analyses}

Preparation of total lipid extracts (TLEs) and study of lipid classes, determination of oil content and acyl composition in seeds of WT and the AS11, lpcat1 and lpcat 2 mutant lines and progeny from crosses were performed as described previously [26,36]. In all cases, the data represent the averages of three-five determinations. 


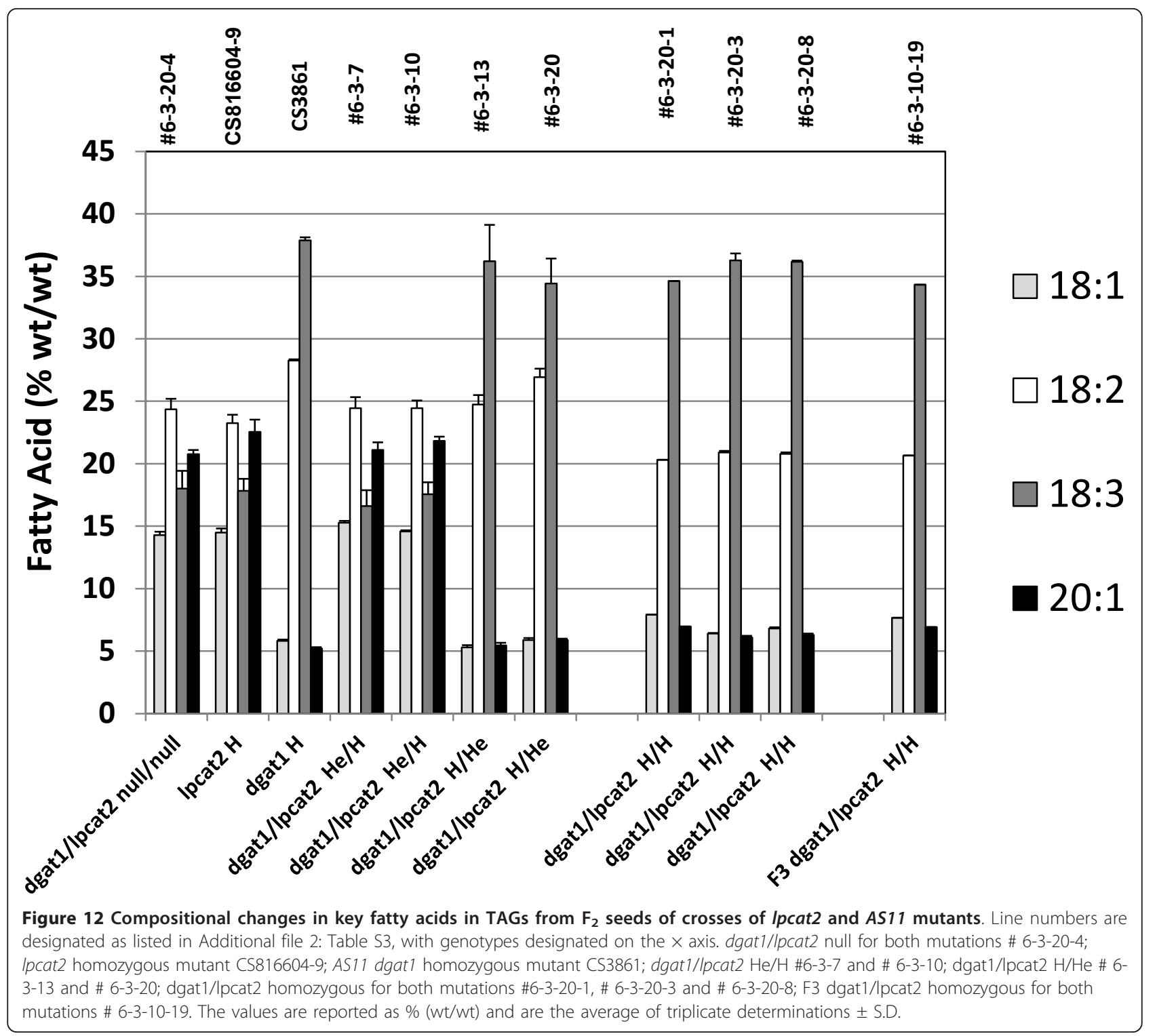

Preparation of Arabidopsis protein fractions

In general, enzyme preparations were made from 200 Arabidopsis siliques of AS11 and WT containing midgreen developing seeds (stages 7-8 as defined above), and immediately powdered with liquid nitrogen in a mortar and pestle. Grinding medium (100 mM HEPES$\mathrm{KOH}, \mathrm{pH} 7.4$ containing $0.32 \mathrm{M}$ Sucrose, $1 \mathrm{mM}$ EDTA, and $1 \mathrm{mM}$ dithiothreitol; $8 \mathrm{~mL} / 50$ siliques) along with $65 \mathrm{mg}$ polyvinylpolypyrrolidone was immediately added, and grinding continued on ice for $5 \mathrm{~min}$. The slurried cell-free homogenate was filtered through two layers of Miracloth (Calbiochem, La Jolla, CA), centrifuged at $3,000 \times g$ for $5 \mathrm{~min}$, the pellet discarded and the supernatant re-centrifuged at $15,000 \times g$ for $30 \mathrm{~min}$. The supernatant was re-centrifuged at $100,000 \times g$ for $1 \mathrm{~h}$ and the resultant pellet was resuspended in $2 \mathrm{~mL}$ of grinding medium, probe-sonicated on ice for $30 \mathrm{~s}$ and protein concentrations were determined using BioRad ${ }^{\mathrm{TM}}$ reagent based on the method of Bradford [37]. Protein concentrations were normalized to the same value for WT and AS11 in each experiment.

\section{Assay of TAG synthesis activity in Arabidopsis mid- developing seeds}

TAG assembly assays were conducted at $\mathrm{pH} 7.4$, with shaking at $100 \mathrm{rev} / \mathrm{min}$ in a water bath at $30^{\circ} \mathrm{C}$ for 60 min. Assay mixtures $(500 \mu \mathrm{L}$ final volume) contained 100-300 $\mu \mathrm{g}$ protein normalized as described above, 90 mM HEPES-NaOH, 0.5 mM ATP, 0.5 mM CoASH, 1 $\mathrm{mM} \mathrm{MgCl} 2$ in the presence of $100 \mu \mathrm{M}\left[1-{ }^{14} \mathrm{C}\right] s n-1,2$ diolein in $0.02 \%$ Tween-20 (specific activity $10 \mathrm{nCi} /$ nmol; pre-purified by TLC on $5 \%$ borate silica G plates) 

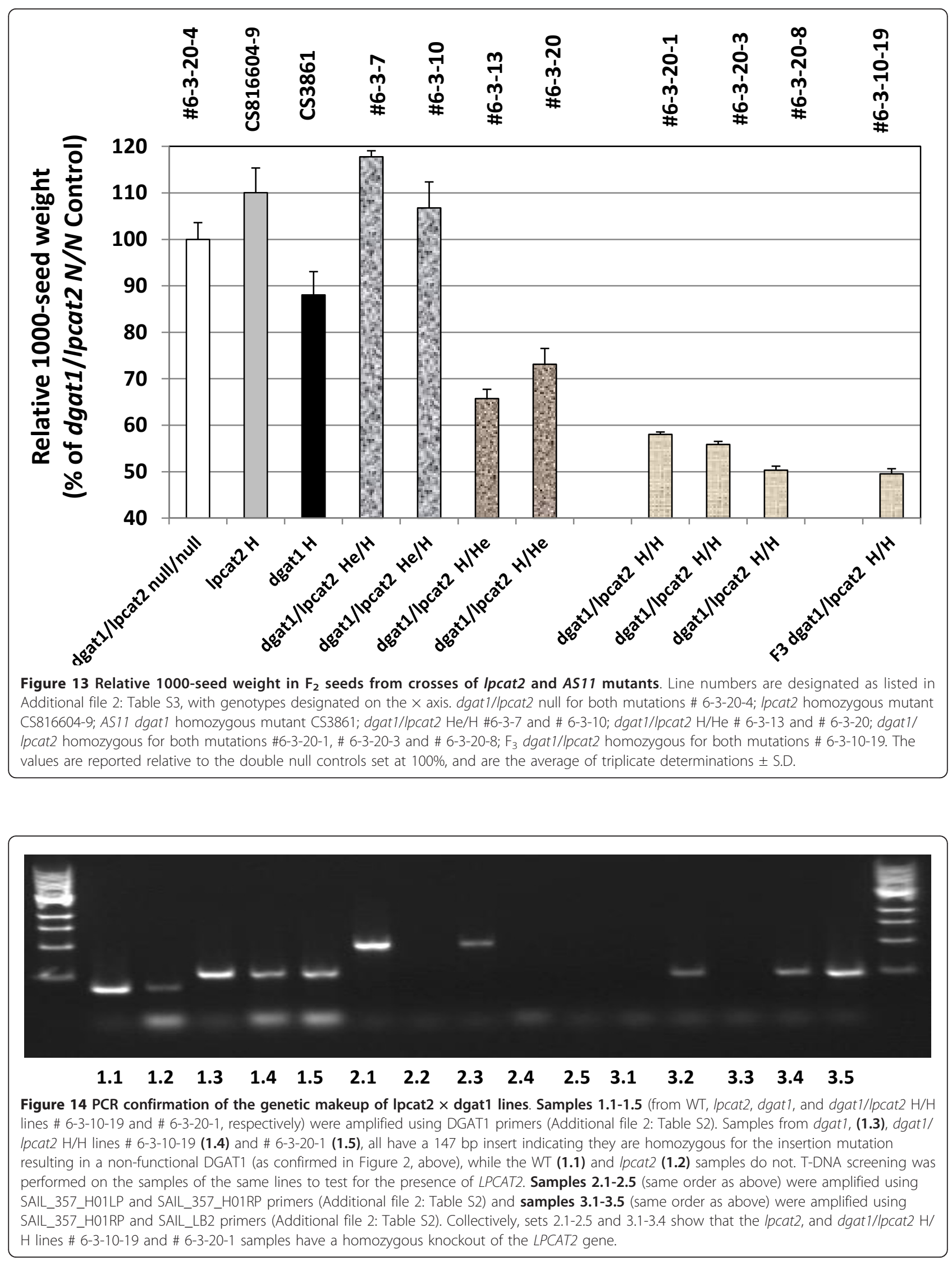


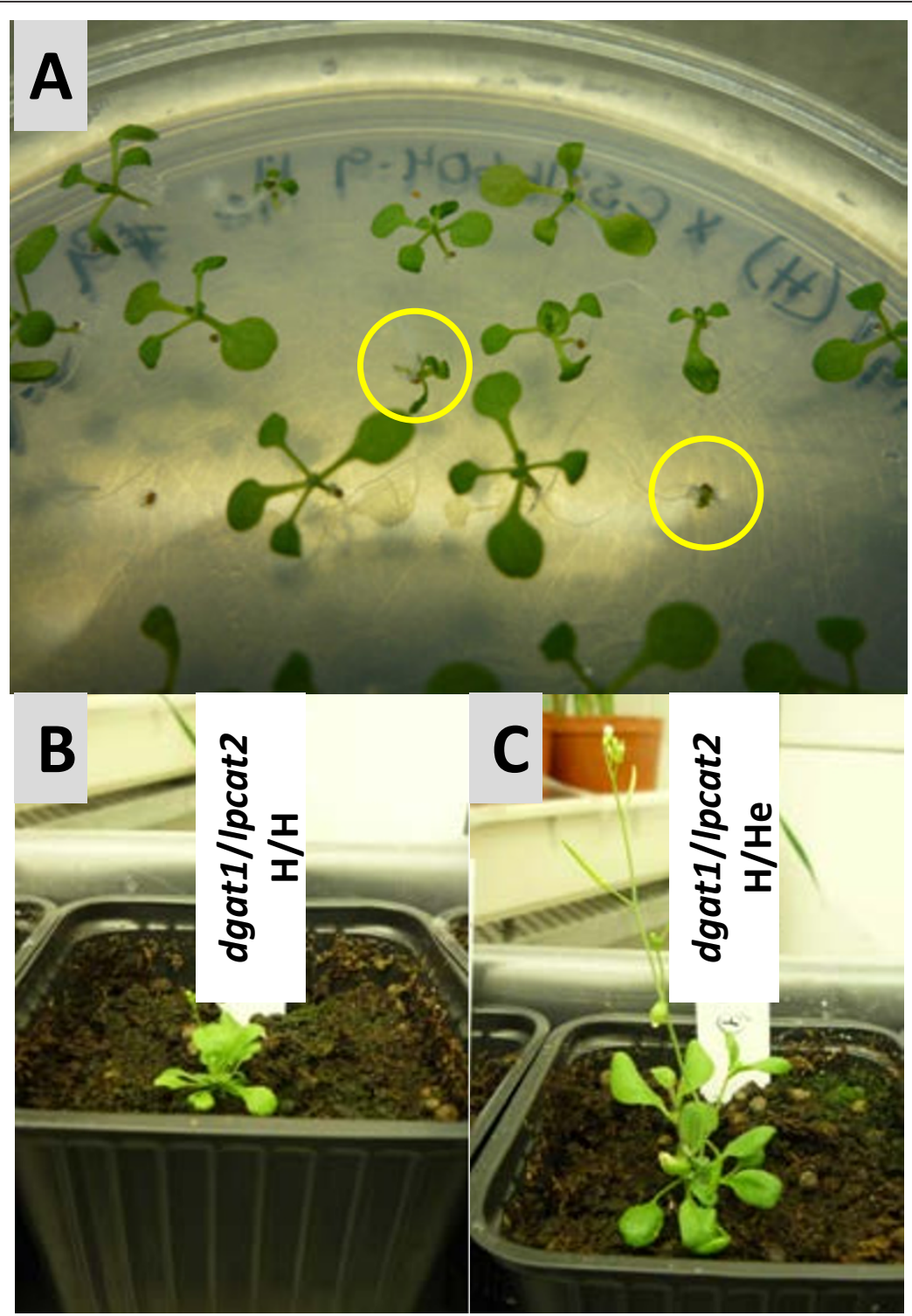

Figure 15 (A) Segregating populations from crosses of dgat1 with Ipcat2. All lines are homozygous for the dgat1 mutation and homozygous, heterozygous or null for Ipcat2. The dgat1/lpcat2 H/H seedlings are circled, showing slow development. (B) Once transferred to soil, bolting is delayed in the dgat 1/lpcat2 $\mathrm{H} / \mathrm{H}$ seedlings. In contrast (C) dgat1/lpcat2 $\mathrm{H} /$ He seedlings develop and bolt normally.

and $18 \mu \mathrm{M}$ unlabeled 18:1-CoA. Reactions were stopped using Isopropanol: $\mathrm{CH}_{2} \mathrm{Cl}_{2}(2: 1:) \mathrm{v} / \mathrm{v}$, and the TLE prepared as described previously [26,36]. The ${ }^{14} \mathrm{C}$-labeled products were resolved by TLC on silica gel G plates developed in hexane: diethyl ether: acetic acid (70:30:1 $\mathrm{v} / \mathrm{v} / \mathrm{v})$, the ${ }^{14} \mathrm{C}$-triolein band visualized on a Bioscan AR-2000 radio-TLC scanner using Win-Scan $2 D^{\odot}$ software (Bioscan Inc., Washington DC) and the band scraped and quantified on a scintillation counter.

\section{Expression of mutated AtDGAT1 in yeast}

The mutated AtDGAT1 (from AS11 as well as A7) in pYES2.1/NT B plasmid were transformed into a quadruple yeast mutant $H 1246 M A T \alpha$ [27] using the S.c. EasyComp ${ }^{\text {TMT}}$ Transformation Kit (Invitrogen, San Francisco, CA). Yeast cells transformed with pYES2.1/NT B plasmid containing the WT DGAT1 or with empty plasmid were used as positive or negative controls, respectively. Transformants were selected by growth on synthetic complete medium lacking uracil (SC-ura), supplemented with $2 \%(\mathrm{w} / \mathrm{v})$ glucose. The colonies were transferred into liquid SC-ura with $2 \%(\mathrm{w} / \mathrm{v})$ glucose and grown at $30^{\circ} \mathrm{C}$ overnight. The overnight culture was diluted to an OD 0.4 in induction medium (SC-ura + $2 \%$ Galactose $+1 \%$ Raffinose), and were induced for $24 \mathrm{~h}$ at $30^{\circ} \mathrm{C}$. The yeast cells were collected and broken with 


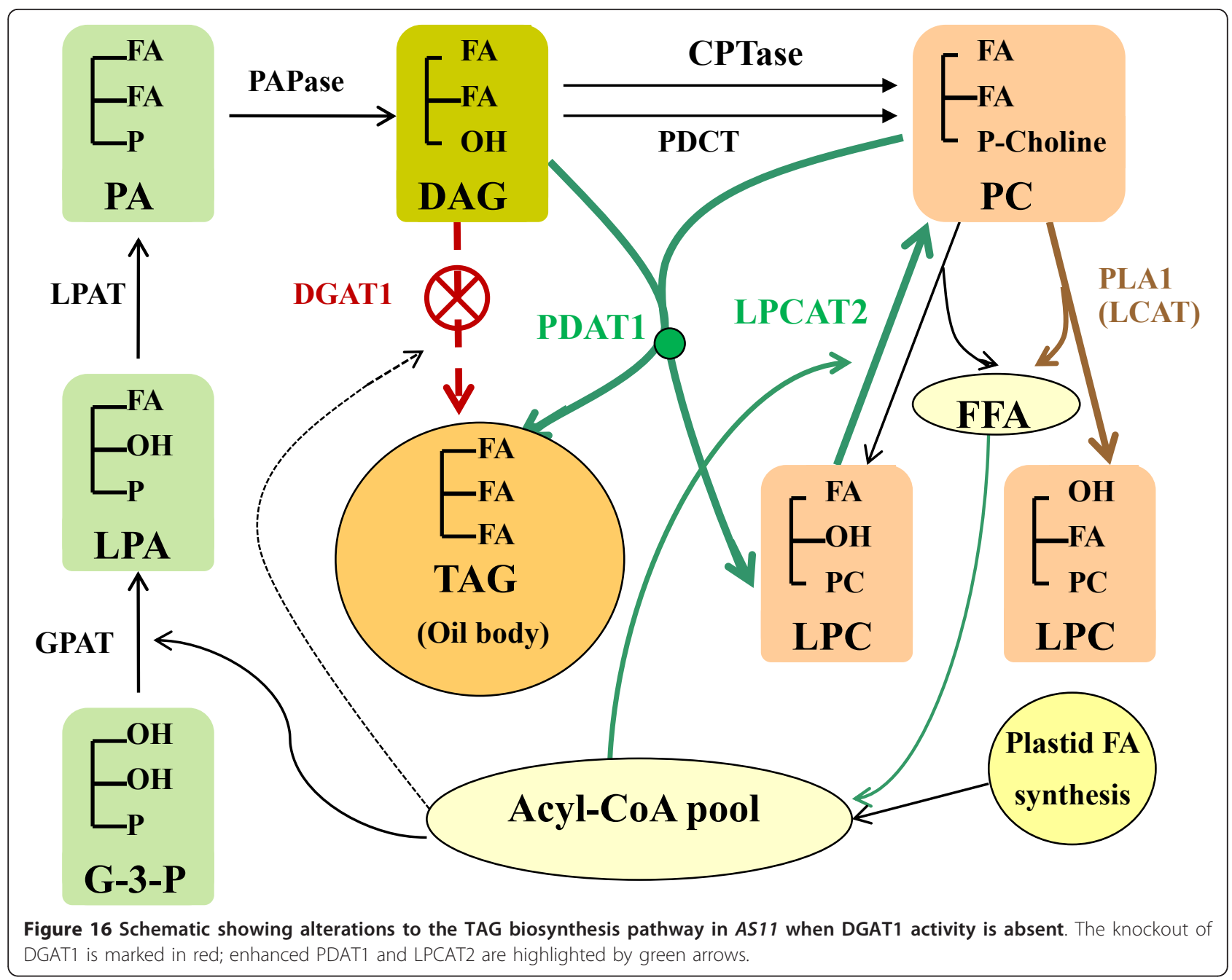

glass beads using a Beadbeater ${ }^{\mathrm{TM}}$. The protein concentrations of the yeast cell lysates were normalized using the Biorad $^{\mathrm{TM}}$ assay and assayed for DGAT activity. DGAT assays were conducted at $\mathrm{pH} 7.4$, with shaking at 100 $\mathrm{rev} / \mathrm{min}$ in a water bath at $30^{\circ} \mathrm{C}$ for $60 \mathrm{~min}$. Assay mixtures $(500 \mu \mathrm{L}$ final volume) contained $100 \mu \mathrm{g}$ of lysate protein, $90 \mathrm{mM}$ HEPES-NaOH, $100 \mu \mathrm{M} s n-1,2$ diolein or $s n-1,2$ dierucin (pre-purified by TLC on $10 \%$ borate silica $\mathrm{H}$ plates and emulsified in $0.02 \%$ Tween-20), and $18 \mu \mathrm{M}$ $1-{ }^{14} \mathrm{C} 18: 1-\mathrm{CoA}$ (specific activity $10 \mathrm{nCi} / \mathrm{nmol}$ ) as the acyl donor. Reactions were stopped using isopropanol: $\mathrm{CH}_{2} \mathrm{Cl}_{2}$ (2:1:) v/v, and extraction of the lipid fraction performed as described previously [26,36]. The ${ }^{14} \mathrm{C}$-labeled TAGs were resolved by TLC on silica gel G plates developed in hexane: diethyl ether: acetic acid (70:30:1 v/v/v), the ${ }^{14} \mathrm{C}$-triolein band visualized on a Bioscan AR-2000 radio-TLC scanner using Win-Scan $2 \mathrm{D}^{\odot}$ software (Bioscan Inc., Washington DC) and the band scraped and quantified on a scintillation counter.

\section{Immunodetection}

The yeast cell lysates were run on a $10 \%$ Tris-HCl SDSPAGE gel, the proteins were then transferred to a nitrocellulose membrane (Nitrobind, Thermo Fisher Scientific, Waltham, MA). The membrane was blocked in PBST (phosphate buffered saline containing 0.5\% Tween 20) containing $4 \%$ skim milk for $60 \mathrm{~min}$, and then incubated with the primary antibody, Anti-Xpress (epitopetagged) antibody (Invitrogen, San Francisco, CA) diluted to $1: 5000$ with PBST containing $2 \%$ skim milk, for 60 min. The membrane was submitted to three washes with PBST followed by three washes with PBS to remove any unbound antibody. Next, the membrane was incubated with a goat anti-mouse IgG peroxidase antibody (Sigma-Aldrich, A2554, St. Louis, MO), diluted to $1: 5000$ with PBST containing $2 \%$ skim milk, for 60 min. The membrane was washed three times with PBST followed by three times with PBS, then the proteins were detected using the Amersham ECL Plus Western 
Blotting Detection Kit (GE Healthcare Life Sciences) with a 10 min exposure time.

\section{LPCAT assays}

LPCAT assays were conducted at $\mathrm{pH} 7.4$, with shaking at $100 \mathrm{rev} / \mathrm{min}$ in a water bath at $30^{\circ} \mathrm{C}$ in a 20 -minute time course. Assay mixtures (500 $\mu \mathrm{L}$ final volume) contained $300 \mu \mathrm{g}$ protein normalized as described above, 90 mM HEPES-NaOH, $0.5 \mathrm{mM}$ ATP, $0.5 \mathrm{mM}$ CoASH, 1 $\mathrm{mM} \mathrm{MgCl} 2$ in the presence of $6 \mu \mathrm{M} \mathrm{L}$ - $\alpha$-palmitoyl- [1${ }^{3} \mathrm{H}$ methyl] lyso-3-phosphatidylcholine (specific activity $60 \mu \mathrm{Ci} / \mathrm{nmol} ; 1.48-2.22 \mathrm{TBq} / \mathrm{mmol})$ and $18 \mu \mathrm{M}\left[1-{ }^{14} \mathrm{C}\right]$ 18:1-CoA (specific activity $10 \mathrm{nCi} / \mathrm{nmol} ; 0.37 \mathrm{GBq} /$ $\mathrm{mmol})$. Reactions were stopped at each time point by adding isopropanol: $\mathrm{CH}_{2} \mathrm{Cl}_{2}(2: 1:) \mathrm{v} / \mathrm{v}$, and the TLE prepared as described previously [26,36]. The ${ }^{14} \mathrm{C}$ and $3 \mathrm{H}-$ labeled products were resolved by 3D TLC performed as follows: The first 2 dimensions were run as described by Yokoyama et al. [38]; 1st D: $\mathrm{CHCl}_{3}: \mathrm{MeOH}$ : Formic Acid (88\%): $\mathrm{H}_{2} \mathrm{O}$ 60: 30: 9 : 2; 2nd D: $\mathrm{CHCl}_{3}: \mathrm{MeOH}$ : Ammonia solution (28\%): $\mathrm{H}_{2} \mathrm{O}$ 50: 40: 7: 3; 3D: The 3 rd D: $100 \%$ ethyl ether. Radiolabeled spots corresponding to standards of LPC and PC were identified using a radio-TLC scanner and the bands scraped and counted on a scintillation counter with a dual ${ }^{3} \mathrm{H} /{ }^{14} \mathrm{C}$ isotope measurement program.

\section{In vivo feeding experiments}

Twenty bolted stems with mid-developing siliques of both WT and AS11 plants were harvested under water and then immediately placed in a solution containing 1 $\mu \mathrm{Ci}$ of ${ }^{14} \mathrm{C}$ sodium acetate in $100 \mu \mathrm{L}$ of water and incubated at room temperature in a fume hood. Once most of this solution was taken up, the plants were supplied with equal aliquots of distilled water during the chase period. At 0, 7, 24 and 30 h, 5 bolted stems were harvested, siliques counted and weighed and then ground with a polytron and a TLE extraction performed as described by Taylor et al. [26]. The lipid extract was resuspended in $1 \mathrm{~mL} \mathrm{CHCl} \mathrm{CHeOH}_{3}: 1.10 \mu \mathrm{L}$ of the solution was counted and the remainder spotted on TLC plates, 3D TLC performed, radiolabeled spots resolved on a radio-TLC scanner, silica bands scraped and radioactivity measured with a scintillation counter.

\section{qRT-PCR comparison of AS11 and WT expression of key lipid genes}

Triplicate biological samples of total RNA were extracted using the method of Wang and Vodkin [39] from Arabidopsis siliques of AS11 and WT containing mid-green developing seeds (stages 7-8 as defined above) and used for qRT-PCR [40]. One microgram of total RNA was reverse-transcribed using SuperScript ${ }^{\circledR}$ II
Reverse Transcriptase (Invitrogen) according to the manufacturer's protocol. The resulting cDNA was then amplified by PCR using Taq DNA polymerase (Invitrogen). PCR conditions comprised an initial cycle of $94^{\circ} \mathrm{C}$ for $3 \mathrm{~min}$; followed by 30 cycles of $94^{\circ} \mathrm{C}$ for $30 \mathrm{~s}, 56^{\circ} \mathrm{C}$ for $30 \mathrm{~s}$, and $72^{\circ} \mathrm{C}$ for $1 \mathrm{~min}$; then $72^{\circ} \mathrm{C}$ for $10 \mathrm{~min}$ to complete the reaction. Concentrations for the individual samples within each group (AS11 and WT) were normalized using $18 \mathrm{~S}$ rRNA levels. The gene-specific primers (listed in Additional file 2: Table S1) were designed according to the target gene sequences as annotated in Genbank ${ }^{\mathrm{TM}}$ (http://www.ncbi.nlm.nih.gov/ genbank/). Amplified RT-PCR products were resolved by electrophoresis on $1 \%$ agarose gels and gel photos taken. The densities of the PCR bands were analyzed using ImageJ image analysis software (National Institutes of Health, USA http://rsbweb.nih.gov/nih-image/) and quantified relative to the "housekeeping gene" $18 \mathrm{~S}$ rRNA transcript signal. Then the ratio of AS11:WT gene expression level was calculated. Specifically, for each gene of interest, first we calculated the ratio of AS11 density/18S rRNA density (= "AS11 ratio") and WT density/18S rRNA density (= "WT ratio"). The difference in specific gene expression in AS11 vs. WT, was then calculated as ("AS11 ratio")/("WT ratio") = V. If V $>1.0$, then the gene is up-regulated in AS11 relative to WT; if $\mathrm{V}<1.0$, then the gene is down-regulated in AS11 relative to $\mathrm{WT}$. The \% relative increase or decrease is then $=([\mathbf{V} \times 100]-100)$.

\section{Affymetrix microarray analysis}

The same triplicate biological samples prepared for the qRT-PCR study (above) were sub-sampled for microarray analysis. Affymetrix microarray hybridizations using the Ath1 whole genome array, containing probe sets representing $\sim 22,800$ genes, were performed using three biological replicate samples for each genotype. Labeling, hybridization, and scanning were performed by the Affymetrix Gene Chip Facility at the University of Toronto (http://www.csb.utoronto.ca/resources/facilities/affymetrix-genechip). Data analysis was performed using GeneSpring ${ }^{\text {TM }}$ software version 7.2. To identify key lipid genes that were differentially expressed between the two genotypes, a per-gene normalization was applied to the values and a parametric test was performed. Genes that exhibited a false discovery rate of $p<0.05$ and passed the minimum signal and fold-change threshold were determined to be differentially expressed. Reported candidate genes were selected by comparing those listed by Beisson et al. [33] with our microarray and qRT-PCR data. The "fold change" was converted to \% change. For the group of genes detailed in Figure 3, the final changes ranged from $-60 \%$ to $+150 \%$. 


\section{Mutant crosses}

\section{Confirmation of Ipcat1 and Ipcat2 T-DNA insertion} mutation lines

Arabidopsis insertion mutation lines [41] SALK_123480 putative for LPCAT1 (At1g12640) and SAIL_357_H01 putative for LPCAT2 (At1g63050) were identified in the Salk Institute T-DNA insertion library database (http:// signal.salk.edu/cgi-bin/tdnaexpress), and seeds were obtained from the Nottingham Arabidopsis Stock Centre (University of Nottingham, UK). According to annotation in the database SALK_123480 contains a T-DNA insertion in the middle of the 7th exon of the LPCAT1 gene and SAIL_357_H01 a T-DNA insertion in the 8th exon of the $L P C A T 2$ gene. Individual plants homozygous for a T-DNA insertion in each the LPCAT1 or LPCAT2 genes were identified by PCR screening using primers SALK_123480LP, SALK_123480RP and SALK_LBb1 (Additional file 2: Table S2) for LPCAT1 and SAIL_357_H01LP, SAIL_357_H01RP and SAIL_LB2 (Additional file 2: Table S2) for LPCAT2. Individual plants from each mutant line lacking a T-DNA insertion in the LPCAT1 or LPCAT2 genes (null segregants) were also identified in the same PCR primer set. Annotation of lines from each set of crosses is as designated in Additional file 2: Table S3.

\section{Transcript analyses of Ipcat1, Ipcat2, dgat1 mutant lines and crosses}

\section{Ipcat1 (See Figure 4)}

Total RNA was isolated from developing siliques from control and SALK_12340 T-DNA insertion mutation plants using CONCERT Plant RNA Reagent (Invitrogen, San Francisco, CA) according to the technical specifications given by the manufacturer. The RNA was then DNAse-treated with TURBO ${ }^{\mathrm{TM}}$ DNase (Ambion, Huntingdon, UK) ) and used (1 mg) for synthesize first strand cDNA with Maxima First Strand cDNA Synthesis Kit (Fermentas.Thermo Fisher Scientific, St. Leon-Rot, Germany). The cDNA was amplified by PCR using Taq DNA Polymerase (Sigma-Aldrich, St. Louis, MO) and the primers SALK_12640-1 s and SALK_12640-6r as listed in Additional file 2: Table S2. PCR conditions comprised an initial cycle of $94^{\circ} \mathrm{C}$ for $1 \mathrm{~min}$; followed by 40 cycles of $94^{\circ} \mathrm{C}$ for $20 \mathrm{~s}, 64^{\circ} \mathrm{C}$ for $20 \mathrm{~s}$, and $72^{\circ} \mathrm{C}$ for $35 \mathrm{~s}$; then $72^{\circ} \mathrm{C}$ for $8 \mathrm{~min}$ to complete the reaction. PCR samples were separated on $1 \%$ agarose gel using $\lambda$-DNA digested with PstI as size marker.

\section{Ipcat2 and AS11 dgat1 (See Figure 5)}

Total RNA was isolated from developing siliques from WT control, AS11(dgat1), SAIL_357_H01 insertion mutation (lpcat2) and AS11/lpcat2 plants by the method of Wang and Vodkin [39]. One microgram of total RNA was reverse-transcribed using SuperScript ${ }^{\circledR}$ II Reverse
Transcriptase (Invitrogen) according to the manufacturer's protocol. The resulting cDNA was then amplified by PCR using Taq DNA polymerase (Invitrogen). PCR conditions comprised an initial cycle of $94^{\circ} \mathrm{C}$ for $3 \mathrm{~min}$; followed by 30 cycles of $94^{\circ} \mathrm{C}$ for $30 \mathrm{~s}, 56^{\circ} \mathrm{C}$ for $30 \mathrm{~s}$, and $72^{\circ} \mathrm{C}$ for $1 \mathrm{~min}$; then $72^{\circ} \mathrm{C}$ for $10 \mathrm{~min}$ to complete the reaction. Concentrations for the individual samples within each group (AS11 and WT) were normalized using $18 \mathrm{~S}$ rRNA levels. Gene specific primers spanning the T-DNA insertional region were used to check for gene transcription. For DGAT1 transcript analysis in lines of WT, the AS11 (dgat1) mutant, lpcat2 mutant and crosses of lpcat 2 with dgat1, the primer pairs were TAG1-mut-primerA and TAG1-mut-primerB. For LPCAT2 transcript analysis in these same lines, the primer pairs were LPCAT2-F2 and LPCAT2-R2 as listed in Additional file 2: Table S2.

\section{Creating double mutants of dgat $1 \times$ Ipcat 1 and dgat $1 \times$ Ipcat2}

Crosses between the AS11 mutant and lpcat1 and lpcat2 T-DNA insertion mutation lines, respectively, were made and $\mathrm{F}_{1}$ plants heterozygous for AS11 and the insertion mutations were identified by PCR using primers listed in Additional file 2: Table S2. $\mathrm{F}_{2}$ seeds segregating for the mutations were planted and screened by PCR. All PCR screening was done as above for LPCAT1 and LPCAT2 and for the AS11 mutation vs WT using primers dgat1-mut-primerA and dgat1-mut-primerB (Additional file 2: Table S2), as designed by Zou et al. [7]. Identification of individuals homozygous for both the AS11 mutation and lpcat2 insertion mutation was only possible after growing a segregating $\mathrm{F}_{2}$ seed population (homozygous for AS11 and heterozygous for lpcat 2 mutant) on agar media containing a $1 / 3$ strength MS and 1\% sucrose (Additional file 2: Table 3).

\section{Additional material}

Additional file 1: Figure S1 Comparison of growth phenotypes of WT,
AS11 and A7 Arabidopsis thaliana lines. While the WT has bolted, both
AS11 and A7 show a delay in plant development: the generative
(reproductive) phase is delayed about 1 week (33\% longer than WT to
mature). Figure S2. (A) Photos of developing siliques from $F_{2}$ dgat1/
Ipcat2 He/H lines \#6-3-7 (left) and \# 6-3-10 (right) showing the many
gaps due to non-fertilized ovules. (B) Photos 1-4: Close-ups of
developing siliques of line \#6-3-10 with arrows pointing to non-fertilized
ovules (photos 1 \& 2). Figure S3. Photos of developing siliques from F 2
dgat1/Ipcat2 H/He line \#6-3-13 showing normal seed development and
pattern.
Additional file 2: Table S1 Primers used for semi-quantitative RT-PCR
conducted in this study. Table S2. Primers used to screen the AS11 dgat1
EMS mutant and Ipcat1 and Ipcat2 T-DNA insertion mutant lines by PCR.
Table S3. Details on crosses performed to develop lines homozygous for
both dgat1 and Ipcat1 mutations or for both dgat1 and Ipcat2 mutations.




\section{Acknowledgements}

This work was partially supported by a grant to D.C.T. from Genome Canada: Genome Prairie Phase II and by funding to A.S.C. from FORMAS (The Swedish Research Council for Environment, Agricultural Sciences and Spatial Planning). The authors also acknowledge, with thanks, the kind assistance of Drs. Mark Smith and Alison Ferrie for their careful reviews of the manuscript and Dr. John Ohlrogge for helpful discussions during the course of this work. This is National Research Council of Canada Publication No. 50191. We thank the Salk Institute Genomic Analysis Laboratory for providing the sequence-indexed Arabidopsis T-DNA insertion mutant and the Nottingham Arabidopsis Stock Centre for providing seeds. Funding for the SIGNAL indexed insertion mutant collection was provided by the National Science Foundation

\section{Author details}

'National Research Council of Canada, Plant Biotechnology Institute, 110 Gymnasium Place, Saskatoon, SK S7N OW9, Canada. ${ }^{2}$ Plant Breeding and Biotechnology, Swedish University of Agricultural Sciences, Box 101, Sundsvägen 14, 23053 Alnarp, Sweden. ${ }^{3}$ College of Agronomy, Northwest A \& F University, No.3 Taicheng Road, Yangling, Shanxi 712100, China. ${ }^{4}$ NRC Plant Biotechnology Institute, 110 Gymnasium Place, Saskatoon, SK S7N 0W9, Canada.

\section{Authors' contributions}

JX performed the GRT-PCR of lipid gene expression in AS11 and WT and assisted in the design of this study, ASC carried out the crosses of the Ipcat mutants with AS11 and selection of the lines with heterozygous or homozygous mutation conditions, TF carried out the microarray analyses of AS11 and WT gene sets and the ectopic expression of the mutated AS11 and A7 DGAT1 proteins in yeast mutant H1246MATa, MZ carried out the work demonstrating the dominant role of PDAT1 in Arabidopsis TAG assembly and provided advice regarding the analyses of the mutant crosses, TH and MG performed the LPCAT and DGAT assays as well as the oil content, seed weight and acyl profiling of the mutant crosses. DCT conceived of the study, led its design and coordination and drafted the manuscript. All authors read and approved the final manuscript.

Received: 21 July 2011 Accepted: 10 January 2012

Published: 10 January 2012

\section{References}

1. Stymne S, Stobart AK: Triacylglycerol biosynthesis. In The biochemistry of plants. Lipids: structure and funchtion. Volume 9. Edited by: Stumpf PK. New York: Academic Press; 1987:175-214.

2. Murphy DJ, Vance J: Mechanisms of lipid-body formation. Trends Biochem Sci 1999, 24:109-115.

3. Wolters-Arts M, Lush WM, Mariani C: Lipids are required for directional pollen-tube growth. Nature 1998, 392:818-821.

4. Zheng Z, Xia Q, Dauk M, Shen W, Selvaraj G, Zou J: Arabidopsis AtGPAT1, a member of the membrane-bound glycerol-3-phosphate acyltransferase gene family, is essential for tapetum differentiation and male fertility. Plant Cell 2003, 15:1872-1887.

5. Cases S, Smith SJ, Zheng YW, Myers HM, Lear SR, Sande E, Novak S, Collins C, Welch CB, Lusis AJ, Erickson S, Farese RV Jr: Identification of a gene encoding an acyl CoA: diacylglycerol acyltransferase, a key enzyme in triacylglycerol synthesis. Proc Natl Acad Sci USA 1998, 95:13018-13023.

6. Katavic V, Reed DW, Taylor DC, Giblin EM, Barton DL, Zou J, MacKenzie SL, Covello PS, Kunst L: Alteration of seed fatty acid composition by an ethyl methanesulfonateinduced mutation in arabidopsis thaliana affecting diacylglycerol acyltransferase activity. Plant Physiol 1995, 108:399-409.

7. Zou J, Wei Y, Jako C, Kumar A, Selvaraj G, Taylor DC: The Arabidopsis thaliana TAG1 mutant has a mutation in a diacylglycerol acyltransferase gene. Plant J 1999, 19:645-653.

8. Hobbs DH, Lu C, Hills MJ: Cloning of a cDNA encoding diacylglycerol acyltransferase from Arabidopsis thaliana and its functional expression. FEBS 1999, 452:145-149.

9. Routaboul JM, Benning C, Bechtold N, Caboche M, Lepiniec L: The TAG1 locus of Arabidopsis encodes for a diacylglycerol acyltransferase. Plant Physiol and Biochem 1999, 37:831-840.

10. Jako C, Kumar A, Wei Y, Zou J, Barton DL, Giblin EM, Covello PS, Taylor DC: Seedspecific over-expression of an Arabidopsis cDNA encoding a diacylglycerol acyltransferase enhances seed oil content and seed weight. Plant Physiol 2001, 126:861-874.

11. Weselake RJ, Shah S, Tang M, Quant PA, Snyder CL, Furukawa-Stoffer TL, Zhu W, Taylor DC, Zou J, Kumar A, Hall L, Laroche A, Rakow G, Raney P, Moloney MM, Harwood $\mathrm{J}$ : Metabolic control analysis is helpful for informed genetic manipulation of oilseed rape (Brassica napus) to increase seed oil content. J Exp Bot 2008, 59:3543-3549.

12. Taylor DC, Zhang Y, Kumar A, Francis T, Giblin EM, Barton DL, Ferrie JR, Laroche A, Shah S, Zhu W, Snyder CL, Hall L, Rakow G, Harwood JL, Weselake RJ: Molecular modification of triacylglycerol accumulation under field conditions to produce canola with increased seed oil content. Botany 2009, 87:533-543.

13. Lardizabal KD, Mai JT, Wagner NW, Wyrick A, Voelker T, Hawkins DJ: DGAT2 is a new diacylglycerol acyltransferase gene family: purification, cloning, and expression in insect cells of two polypeptides from Mortierella ramanniana with diacylglycerol acyltransferase activity. J Biol Chem 2001, 276:38862-38869.

14. Cases S, Stone SJ, Zhou P, Yen E, Tom B, Lardizabal KD, Farese RV Jr: Cloning of DGAT2, a second mammalian diacylglycerol acyltransferase, and related family members. J Biol Chem 2001, 276:38870-38876.

15. Shockey JM, Gidda SK, Chapital DC, Kuan JC, Dhanoa PK, Bland JM, Rothstein SJ, Mullen RT, Dyer JM: Tung Tree DGAT1 and DGAT2 have nonredundant functions in triacylglycerol biosynthesis and are localized to different subdomains of the endoplasmic reticulum. Plant Cell 2006, 15:1872-1887.

16. Kroon JT, Wei W, Simon WJ, Slabas AR: Identification and functional expression of a type 2 acyl-CoA: diacylglycerol acyltransferase (DGAT2) in developing castor bean seeds which has high homology to the major triglyceride biosynthetic enzyme of fungi and animals. Phytochemistry 2006, 67:2541-2549.

17. Zhang M, Fan J, Taylor DC, Ohlrogge JB: DGAT1 and PDAT1 acyltransferases have overlapping functions in Arabidopsis triacylglycerol biosynthesis and are essential for normal pollen and seed development. Plant Cell 2009, 21:3885-3901.

18. Dahlqvist A, Stahl U, Lenman M, Banas A, Lee M, Sandager L, Ronne $H$, Stymne S: Phospholipid: diacylglycerol acyltransferase: an enzyme that catalyzes the acyl-CoA-independent formation of triacylglycerol in yeast and plants. Proc Natl Acad Sci USA 2000, 97:6487-6492.

19. Banas A, Dahlqvist A, Stahl U, Lenman M, Stymne S: The involvement of phospholipid: diacylglycerol acyltransferases in triacylglycerol production. Biochem Soc Trans 2000, 28:703-705.

20. Stahl U, Carlsson AS, Lenman M, Dahlqvist A, Huang B, Banas W, Banas A, Stymne S: Cloning and functional characterization of a phospholipid: diacylglycerol acyltransferase from Arabidopsis. Plant Physiol 2004, 135:1324-1335.

21. Mhaske V, Beldjilali K, Ohlrogge J, Pollard M: Isolation and characterization of an Arabidopsis thaliana knockout line for phospholipid: diacylglycerol transacylase gene (At5g13640). Plant Physiol Biochem 2005, 43:413-417.

22. Lu C, de Noyer SB, Hobbs DH, Kang J, Wen Y, Krachtus D, Hills MJ: Expression pattern of diacylglycerol acyltransferase-1, an enzyme involved in triacylglycerol biosynthesis, in Arabidopsis thaliana. Plant Mol Biol 2003, 52:31-41.

23. Kim HU, Lee K-R, Go YS, Jung JH, Suh M-C, Kim JB: Endoplasmic reticulumlocated PDAT1-2 from castor bean enhances hydroxy fatty acid accumulation in transgenic plants. Plant Cell Physiol 2011, 52:983-993.

24. Van Erp H: Castor phospholipid: diacylglycerol acyltransferase facilitates efficient metabolism of hydroxy fatty acids in transgenic Arabidopsis. Plant Physiol 2011, 155:683-693.

25. Stahl U, Stalberg K, Stymne S, Ronne H: A family of eukaryotic lysophospholipid acyltransferases with broad specificity. FEBS Lett 2008, 582:305-309.

26. Taylor DC, Weber N, Hogge LR, Underhill EW, Pomeroy MK: Formation of Trierucoylglycerol (Trierucin) from 1,2-Dierucoylglycerol by a homogenate of microspore-derived embryos of Brassica napus L. J Am Oil Chem Soc 1992, 69:355-358.

27. Sandager L, Gustavsson MH, Stahl U, Dahlqvist A, Wiberg E, Banas A, Lenman M, Ronne H, Stymne S: Storage lipid synthesis is non-essential in yeast. J Biol Chem 2002, 277:6478-6482.

28. Xu J, Francis T, Mietkiewska E, Giblin EM, Barton DL, Zhang Y, Zhang M, Taylor DC: Cloning and characterization of an acyl-CoA-dependent diacylglycerol acyltransferase 1 (DGAT1) gene from Tropaeolum majus, 
and a study of the functional motifs of the DGAT protein using sitedirected mutagenesis to modify enzyme activity and oil content. Plant Biotechnol J 2008, 6:799-818.

29. Zhang M, Fan J, Taylor DC, Ohlrogge JB: DGAT1 and PDAT1

acyltransferases have overlapping functions in Arabidopsis triacylglycerol biosynthesis and are essential for normal pollen and seed development. The Plant Cell 2009, 21:3885-3901.

30. Mañas-Fernández A, Vilches-Ferrón M, Garrido-Cárdenas J, Belarbi E-H, Alonso D, García-Maroto F: Cloning and molecular characterization of the acyl-CoA: diacylglycerol acyltransferase 1 (DGAT1) Gene from Echium. Lipids 2009, 44:555-568.

31. Guihéneuf F, Leu S, Zarka A, Khozin-Goldberg I, Khalilov I, Boussibab S: Cloning and molecular characterization of a novel acyl-CoA: diacylglycerol acyltransferase 1-like gene (PtDGAT1) from the diatom Phaeodactylum tricornutum. FEBS J 2011, 278:3651-3666.

32. Turchetto-Zolet AC, Maraschin FS, de Morais GL, Cagliari A, Andrade CMB, Margis-Pinheiro M, Margis R: Evolutionary view of acyl-CoA diacylglycerol acyltransferase (DGAT), a key enzyme in neutral lipid biosynthesis. BMC Evolutionary Biology 2011, 11:263, doi:10.1186/1471-2148-11-263.

33. Beisson F, Koo AJK, Ruuska S, Schwender J, Pollard M, Thelen JJ, Paddock T, Salas JJ, Savage L, Milcamps A, Mhaske VB, Cho Y, Ohlrogge JB: Arabidopsis genes involved in acyl lipid metabolism a 2003 census of the candidates, a study of the distribution of expressed sequence tags in organs, and a web-based database. Plant Physiol 2003, 132:681-697.

34. Shen W, Chen Q, Wang L, Zou J: Functional Study of Lysophosphatidylcholine Acyltransferase in Arabidopsis. Proceedings of the 18th International Symposium on Plant Lipids Bordeaux; 2008, 102.

35. Lu C, Xin Z, Rin Z, Miquel M, Browse J: An enzyme regulating triacylglycerol composition is encoded by the ROD1 gene of Arabidopsis. Proc Nat'l Acad Sci USA 2009, 106:18837-18842.

36. Taylor DC, Barton DL, Rioux KP, Mackenzie SL, Reed DW, Underhill EW, Pomeroy MK, Weber N: Biosynthesis of acyl lipids containing very long chain fatty acids in microspore-derived and zygotic embryos of Brassica napus L cv Reston. Plant Physiol 1992, 99:1609-1618.

37. Bradford MM: A rapid and sensitive method for the quantification of microgram quantities of protein utilizing the principle of protein-dye binding. Anal Biochem 1976, 72:248-254.

38. Yokoyama K, Shimiza F, Setaka M: Simultaneous separation of lysophospholipids from the total lipid fraction of crude biological samples using two-dimensional thinlayer chromatography. J Lip Res 2000, 41:142-147.

39. Wang CS, Vodkin LO: Extraction of RNA from tissues containing high levels of procyanidins that bind RNA. Plant Mol Bio Rep 1994, 12:132-145.

40. Meadus WJ: A semi-quantitative RT-PCR method to measure the in vivo effect of dietary conjugated linoleic acid on porcine muscle PPAR gene expression. Biol Prod Online 2003, 5:20-28.

41. Alonso JM, Stepanova AN, Leisse TJ, Kim CJ, Chen H, Shinn P, Stevenson DK, Zimmerman J, Barajas P, Cheuk R, Gadrinab C, Heller C, Jeske A, Koesema E, Meyers CC, Parker H, Prednis L, Ansari Y, Choy N, Deen H, Geralt M, Hazari N, Hom E, Karnes M, Mulholland C, Ndubaku R, Schmidt I, Guzman P, Aguilar-Henonin L, Schmid M, Weigel D, Carter DE, Marchand T, Risseeuw E, Brogden D, Zeko A, Crosby WL, Berry CC, Ecker JR: Genome-wide insertional mutagenesis of Arabidopsis thaliana. Science 2003, 301:653-657.

doi:10.1186/1471-2229-12-4

Cite this article as: Xu et al:: Triacylglycerol synthesis by PDAT1 in the absence of DGAT1 activity is dependent on re-acylation of LPC by LPCAT2. BMC Plant Biology 2012 12:4.

\section{Submit your next manuscript to BioMed Central and take full advantage of:}

- Convenient online submission

- Thorough peer review

- No space constraints or color figure charges

- Immediate publication on acceptance

- Inclusion in PubMed, CAS, Scopus and Google Scholar

- Research which is freely available for redistribution

Submit your manuscript at www.biomedcentral.com/submit
Biomed Central 